\title{
Research Study On After Sales Services of Gas Turbines
}

\author{
${ }^{1}$ Ravi Raj, ${ }^{2}$ Prof Harsh Dwivedi , \\ ${ }^{I}$ Under the Guidance of MR. Joseph Jeya Anand S Professor, VIT Business School September 2012 \\ ${ }^{2}$ Director Poddar Instituite of Management, Jaipur
}

Certificate: This is to certify that the Project Report titled 'Research Study On After Sales Services Of Gas Turbines' submitted by Ravi Raj, Reg. No $11 \mathrm{mba1044}$ to VIT Business School, VIT University, Vellore in partial fulfillment of the requirements for the degree of Master of Business Administration is a bonafide record of work carried out by him / her under my supervision. The contents of this report, in full or in part have not been submitted in any form to any other institute or university for the award of any degree or diploma

\section{Acknowledgement}

Foremost, I would like to express my sincere gratitude to my advisor Prof. MR. Joseph Jeya Anand $\mathbf{S}$ for the continuous support of my study and research, for his patience, motivation, enthusiasm, and immense knowledge. His guidance helped me in all the time of research and writing of this report. I could not have imagined having a better advisor and mentor for my research study.

Besides my advisor, I would like to thank the rest of my project adviser: Mr. Bimlendu Rajesh (Regional Manager - BGGTS) for his encouragement, insightful comments, and hard questions. My sincere thanks also goes to Mr. Vijay Kumar Tata (General Manager - HR \& Admin- BGGTS), for offering me the summer internship opportunities in their groups and leading me working on diverse exciting projects.I also thank Dr.Kalyani Rangarajan (Director VIT Business School) for her reference, to take me this opportunity to do internship in BGGTS. Last but not the least; I would like to thank my brother who guided me throughout the internship.

\section{Contents}

Table 1.1

\begin{tabular}{|c|c|c|}
\hline \multirow{3}{*}{ CHAPTER } & T I T L E & PAGE \\
NO \\
\hline \multirow{2}{*}{ I } & Introduction to the Industry & $9-13$ \\
& Introduction to the Study & $13-14$ \\
II & Objective of Study & $14-16$ \\
& Hypothesis & $17-18$ \\
III & Organization of the Report & 18 \\
& Review of Literature & $18-23$ \\
& Research Methodology & $23-26$ \\
IV & Data Analysis \& Interpretation & $26-76$ \\
& Analysis of Variance (ANOVA) & $50-62$ \\
V & Correlation Analysis & $50-76$ \\
& Weighted Average Method & $80-84$ \\
& Findings & $77-78$ \\
VI & Suggestions & 78 \\
& Conclusion & $79-85$ \\
& References & 86 \\
& Questionnaire & 8794 \\
\hline
\end{tabular}




\begin{tabular}{|c|c|c|}
\hline \multicolumn{2}{|c|}{ List of Tables \& Charts } & Table:- 1.2 \\
\hline S.NO & T I T L E & PAGE NO \\
\hline Tables & CONTENTS & $5-6$ \\
1.1 & List of Tables \& Charts & 13 \\
1.2 & BGGTS advantages - resources & $17-18$ \\
1.3 & Hypothesis & 20 \\
1.4 & Creating service products 1 & 21 \\
1.5 & Creating service products 2 & 26 \\
1.6 & Reliability Test:- & 50 \\
1.7 & Test for correlation and ANOVA & 50 \\
1.8 & Test for correlation and ANOVA & 51 \\
1.9 & Test for correlation and ANOVA & 52 \\
2 & Test for correlation and ANOVA & 52 \\
2.1 & Test for correlation and ANOVA & 53 \\
2.2 & Test for correlation and ANOVA & 55 \\
2.3 & Test for correlation and ANOVA & 56 \\
2.4 & Test for correlation and ANOVA & 57 \\
2.5 & Test for correlation and ANOVA & 57 \\
2.6 & Test for correlation and ANOVA & 58 \\
2.7 & Test for correlation and ANOVA & 58 \\
2.8 & Test for correlation and ANOVA & 60 \\
2.9 & Test for correlation and ANOVA & 60 \\
3 & Test for correlation and ANOVA & 62 \\
3.1 & Test for correlation and ANOVA & \\
3.3 & & \\
\hline
\end{tabular}

\section{Introduction to the Industry}

The beginning: - Joint venture signed in November, 1997 between BHEL and GE of USA to form a JV company in India to provide After Sales Market support on exclusive basis for all heavy-duty GE design Gas Turbines in India and neighboring countries.

\begin{tabular}{|c|c|c|c|}
\hline \multicolumn{2}{|c|}{ About BHEL: - } & \multicolumn{2}{|c|}{ About GE:- } \\
\hline & India's power major & \# & Most admired company \\
\hline \# & 14 manufacturing units & \# & $\begin{array}{l}\text { World's leading GT } \\
\text { manufacturer }\end{array}$ \\
\hline & Manufactures GE tech. GT & \# & 6 business \\
\hline
\end{tabular}

Background of BHEL :- (BHEL) (BSE: 500103 , NSE: BHEL) is one of the oldest and largest state-owned "engineering" and "manufacturing" enterprise in India in the energy related and infrastructure sectors which include Power, Railways, Transmission and Distribution, Oil and Gas sectors and many more. It is the $12^{\text {th }}$ largest power equipment manufacturer in the world. BHEL is the only engineering company on the list, which contain online retail firm. The company has been earning profits continuously since 1971-72 and paying dividends since 1976-77. 74\% of the total power generated in India is produced by equipment manufactured by BHEL.BHEL electronics division supplies gas turbine system for all ratings (frame sizes) of heavy duty gas turbines, which are manufactured/ supplied by BHEL, Hyderabad. BHEL is the first and the only licensee of GE, USA for manufacturing of Mark-IV control systems and the licensee for Mark-V and Mark-VI control system.

BHEL's gas turbine controls are supplied for mechanical drives, captive power plants (Simple cycle / combined cycle) and co-generation plants. BHEL is also providing technical services, supply of spares and up gradation of control system with the current technologies.

Background of GE: - Over the year there has been one organization that has dominated the global market. General Electrics leads all other firms in this market, consistently maintaining a market share of more than $40 \%$ for many consecutive years. . The main reasons for this have been its brand name and also the large portfolio of gas turbines that GE sells. It offers the widest range of heavy duty gas turbine in the market, ranging from 26 MW to $480 \mathrm{MW}$. It has a global installed fleet of more than 6,000 gas turbines: the largest installed base of any 
gas turbine supplier worldwide. GE has a strong presence in countries such as Saudi Arabia, Nigeria, Spain, Germany, South Africa and Taiwan.

After the economic crisis, the global gas turbine market is currently in revival phase. Very few countries were able to insulate themselves from the liquidity crunch that followed it. This resulted into a considerable decrease in the global gas turbine orders in. The liquidity crunch that followed the subprime crisis resulted in absence of financing options for various projects. The lack of confidence of investors in projects resulted in the overall decline of the market. The global gas turbine market fell from $\$ 19,463.8 \mathrm{~m}$ in 2007 to $\$ 10,727.4 \mathrm{~m}$ in 2009 . This slump clearly indicates the impact that the crisis had on the turbine market. How aver the future of this market looks bright, with many projects scheduled to go live in future and many contracts undertaken in the gas turbine market.

Objectives of joint venture: - Leveraging the best resources of two majors to offer after market services in India and neighbor regions.

BGGTS Experience and expertise:-

- $\quad$ Ranges from parts to services and repairs

- $\quad$ Leveraging on experience of $7000 \mathrm{GE}$ gas turbines worldwide

- Offices located in each region for quick reach

- Total number of fleet in India region including (Northern region, western region, Eastern region and southern region) is 225 .

- $\quad$ Regional offices are located in : Delhi, Kolkata, Vadodara, and Hyderabad

BGGTS Journey: - It started commercial operations in Q-1/1998. All the three important sectors namely parts, services and repairs were done under one roof. Repair facility accredited with ISO 9001, 14001 and OHSAS 18001 in April, 03. It executed several fuel conversion projects, turbine improvement and control retrofit projects. It introduced Mark VI to India in 2004. In present scenarios it is growing with customer's confidence. BGGTS plant Life cycle includes:- [Value to customers ]

$\checkmark$ Customer information \& training

$\checkmark$ Plant improvement \& Engg. advisory services

$\checkmark$ Output planning \& parts planning

$\checkmark$ Supply of spare parts

$\checkmark$ Repair \& inspection services

Project experience of BGGTS:-

$\checkmark 3 \times$ Fr 5 Naphtha fuel conversion for MUL

$\checkmark 2 \times$ Fr 5 Naphtha fuel conversion for BPCL

$\checkmark \quad 1 \times$ Fr 5 fuel gas conversion for IFFCO

$\checkmark \quad 2 \times$ Fr 6 fuel gas conversion for NRL

$\checkmark$ Engineering of mixed fuel firing for NTPC Kawas

$\checkmark$ Engineering of gas fuel firing for IPCL Gandhar

$\checkmark$ Fuel change studies for GIPCL,AECO,RIL

$\checkmark$ Turbine upgrades for AECO, GIPCL, NFCL, L\&T, IOC Mathura, Indogulf, AES Srilanka, IPGCL,PPCL Delhi

$\checkmark$ Rehabilitation of gas turbines for IPGCL, TPAEC, BPCL, RPC, Ultratech, GSEC, BPDB

Outage services :- (Complete outage planning and execution on supervisory or turnkey), (Inspection services for gas turbines, generators, gear boxes, control systems and auxiliaries), (supply of parts), (repair services), (multiyear maintenance programs), (project management services), (Till implementation).

$\checkmark$ Customer plant Mgr.

$\checkmark$ Single source total solutions

$\checkmark$ Parts

$\checkmark$ Repairs

$\checkmark$ Services

$\checkmark$ Technical solutions

$\checkmark$ Single point accountability 
Research Study On After Sales Services of Gas Turbines

BGGTS advantages - resources: - Better equipped to handle customer's emergency

Table 1.3

\begin{tabular}{|l|l|l|}
\hline GE's centralized support & BHEL's support & BGGTS's strength \\
\hline $24 \times$ 7power answer center & complete info on plant design & Experienced TA's \\
\hline GT centers of excellence & Info on aux equipments & Average exp- 12 yrs \\
\hline $\begin{array}{l}\text { Product service engineers/ } \\
\text { Design engineers }\end{array}$ & Info on aux equipments & $\begin{array}{l}\text { Proximity of its regional officers } \\
\text { to customers }\end{array}$ \\
\hline Parts center & In country repair facility & $24 \times$ 7 supports on phone \\
\hline
\end{tabular}

Introduction to the Study:-

Gas Turbine is an internal-combustion engine consisting essentially of an air compressor, combustion chamber, and turbine wheel that is turned by the expanding products of combustion.

This study is relevant for BGGTS to identify the factors that may effect after sales services, and factors that could be effective to make good relationship with the clients, and satisfy them further in the future. Though study is conducted in BGGTS, but the finest support is provided by IPGCL \& PPCL, by responding to the questionnaire supplied to them. The key areas I tried to cover under this project are:-

$\checkmark$ After sales services parameters that are critical for client's plant.

$\checkmark \quad$ Key drivers, for operation and maintenance (O\&M), for clients plant.

$\checkmark$ Operation \& maintenance cost factors, in order of its importance to their plant.

$\checkmark$ Services that need to be improve in the future.

$\checkmark$ To identify the conversion/modification \& upgrade services important for their plant.

$\checkmark \quad$ To identify Preferred decision making factors for clients.

Outcome of this could be implemented effectively to retain and satisfy the loyal as well as prospective customers. To collect the information, questionnaire is being used; I personally made my presence to the respondents for explaining about their queries regarding questions being asked to them through questionnaire. SPSS software is used for interpreting and analyzing the data to come up for the statistical results.

\section{Objective of study:-}

Post-warranty customer support for a products or services

In some of the cases after sales service can be almost as important as the initial purchase. The manufacturer, retailer, or service provider determine what should be included in any guarantee or warranty package. This generally includes the duration of the warrant, traditionally one year from the date of purchase but increasingly two or more years, maintenance or replacement policy, items included/excluded, labor costs, and speed of response. In the case of service provider, post sales service might include additional training or help desk availability.

Customer's perception of the degree of willingness with which, a supplier deal with a question or complaint, speed of response, and action taken is also equally important. This study is based on customer's feedback about the post sales services being offered by an organization that deals with "heavy-duty gas turbine" services. This indicates the factor, on which customer retention is based.

This study indicates that there are various factors on which customer's satisfaction is dependent and once an organization takes care of it; it becomes easy for them to retain already existing customers. From the competitors' point of view it becomes more important to take care of the needs of the customers. This study indicates that how a competitor could be aggressive once it gates the opportunity to serve the customer. BGGTS is the organization which takes care of all those activities that could keep competitors apart from its customer, to accomplish this objective BGGTS provides best services, repairing services, supplying parts\& technical solutions to the customers. BGGTS follows a pattern for workflow of planning its services, \& that includes

A: - Turbine operators request for the services.

$>$ B: - Operators information is forwarded to service marketing / sales, \& there after service engineering is informed for operational experience and frame specific analysis.

$>$ C: - Unit specific information (history of operations old/new) send to service marketing sales and this unit gives the operation information and unit specific information to service engineering team for unit specific service analysis.

$>$ D: - Service recommendation is recommended by service engineering team and then after final bid is done followed by service agreement. 


\section{$>\quad$ Inspirations of objective:-}

Interesting facts on customer retention: - It is claimed by Reichheld and Sasser (1990) that:-

$\checkmark$ Acquiring new customers comprises of five times the cost of retaining existing customers.

$\checkmark$ An average company loses $10 \%$ of its customers each year.

$\checkmark$ Lessening the defection rate by as low as $5 \%$ can increase companies profit by $25 \%$ depending on industries standards.

$\checkmark$ Customer profits keep on increasing over the life of the retained customer as cost in retaining the customer becomes lower and lower

However, Corrol \& Reichheld (1992) dispute these calculations claiming that they result from faulty crosssectional analysis.

Main objectives of study:-

a) The main objective of this study is to recognize the factors that could be helpful to retain the customers.

b) Sustain the customers for their life time value, through well defined services policies, and customer friendly environment.

c) Our objective is to determine the voice of customers, and implement it into the services being offered by BGGTS.

d) Determine the steps, BGGTS could implement to strengthen "relationship / partnership" through services, repair, and part supply of "gas turbines" to the existing customers.

Hypothesis:-

Table :-1.4

\begin{tabular}{|c|c|}
\hline H1 & $\begin{array}{l}\text { There is no significant difference among the association in the age group, }<30,30-45 \&>45 \text {, } \\
\text { respondents in their choice of "Service" as the critical parameter for after sales service for their plant }\end{array}$ \\
\hline $\mathrm{H} 2$ & $\begin{array}{l}\text { There is no significant difference among the association in the age group, }<30,30-45 \&>45 \text {, } \\
\text { respondents in their choice of "Maintenance" as the critical parameter for after sales service for their } \\
\text { plant }\end{array}$ \\
\hline $\mathrm{H} 3$ & $\begin{array}{l}\text { There is no significant difference among the association in the age group, }<30,30-45 \&>45 \text {, } \\
\text { respondents in their choice of "Online support" as the critical parameter for after sales service for their } \\
\text { plant }\end{array}$ \\
\hline $\mathrm{H} 4$ & $\begin{array}{l}\text { There is no significant difference among the association in the age group, }<30,30-45 \&>45 \text {, } \\
\text { respondents in their choice of "High combined cycle efficiency" as the key driver for O\&M(Operation } \\
\& \text { maintenance) for their plant }\end{array}$ \\
\hline H5 & $\begin{array}{l}\text { There is no significant difference among the association in the age group, }<30,30-45 \&>45 \text {, } \\
\text { respondents in their choice of "Low specific cost(per KW)" as the key driver for O\&M(Operation \& } \\
\text { maintenance) for their plant }\end{array}$ \\
\hline H6 & $\begin{array}{l}\text { There is no significant difference among the association in the age group, }<30,30-45 \&>45 \text {, } \\
\text { respondents in their choice of "High availability" as the key driver for O\&M(Operation \& } \\
\text { maintenance) for their plant }\end{array}$ \\
\hline $\mathrm{H} 7$ & $\begin{array}{l}\text { There is no significant difference among the association in the age group, }<30,30-45 \&>45 \text {, } \\
\text { respondents in their choice of "High simple cycle efficiency" as the key driver for O\&M(Operation \& } \\
\text { maintenance) for their plant }\end{array}$ \\
\hline H8 & $\begin{array}{l}\text { There is no significant difference among the association in the age group, }<30,30-45 \&>45 \text {, } \\
\text { respondents in their choice of "Pressure ratio" as the key driver for O\&M(Operation \& maintenance) } \\
\text { for their plant }\end{array}$ \\
\hline
\end{tabular}

\section{Organization of the Report:-}

All the variables used in my research is defined in the variable view. Particulars such as name of variables, data entered should be in string or numeric, cell width for variable data, decimals, detailed label of the variable, value label of the variable, scale on which the variables is measured i.e. Nominal/Ordinal/Scale, these all are defined.

\section{Review of literature:-}

While early gas engine were reliable generator of electricity but because they were big, heavy, inefficient devices modern gas turbines was developed in the late $19^{\text {th }}$ century to replace them. During $19950 \mathrm{~s}$ and 1960s gas turbine continued to dominate an expending power generation market, as fossil fuel prices remained low \& ever large steam turbines were brought on line. In 1986, General Electric's profit of introduced a new series of advance gas-fixed turbine that turned around its faltering power system segment. In 1994, operating profits of its power division business reached \&1.2 billion to \& 5.9 billion in sales. Just one year later profits had fallen about one-third, to $\$ 770$ million, due to several factors in which Design flows, that could lead 
to cracks, was the most predominant. GE had to make one of the biggest and most expensive recalls in the electric power business. In total $22 \mathrm{GE}$ turbines in U.S \& overseas had to be shutdown to be fixed an additional 28 being shipped or installed required retrofitting with new components. GE has also lost some orders to competitors in Asia, including a key contract in China. This made GE to think upon after sales services. And took several steps regarding service up gradations \& because of this GE survived. During the early $20^{\text {th }}$ century, turbine increasingly became international in scope. Major American and European manufacturers strengthen their presence in each other's market, leading to the deadly competitive environment, and hence scope for after sales services increased to satisfy the customers demand and also to retain customers for their life time value. This infect created the need to understand the customers behavior in different cultural and geographical contexts. The 1999 AMR research report from Gartner stated that business earn 45\% of gross profits from the aftermarket, although it accounts for only $24 \%$ of the revenues. Cohen, Agrawal \& Agrawal (2006) also thought that customers not only expect product to be perfect but they do expect manufacturers to fix things quickly, when they breakdown, especially in the industrial machinery industry, customers are more eager to get good performance on after sales-service.[Two chains compared(Cohen, Agrawal \& Agrawal,2006)]

Table:- 1.5

\begin{tabular}{|l|l|l|}
\hline Parameter & Manufacturing supply chain & After sales service supply chain \\
\hline Nature of demand & Predictable, can be forecast & Always unpredictable sporadic \\
\hline Required response & Standard, can be scheduled & ASAP (some day or next day) \\
\hline Number of SKUs & Limited & 15 to 20 times more \\
\hline Product portfolio & Largely homogeneous & Always heterogeneous \\
\hline Delivery network & $\begin{array}{l}\text { Depends on nature of the } \\
\text { products (multiple networks } \\
\text { necessary ) }\end{array}$ & $\begin{array}{l}\text { Single network capability \& } \\
\text { capable of delivering different } \\
\text { service product }\end{array}$ \\
\hline Inventory management aim & Maximize velocity of resources & Pre position resources \\
\hline Reverse logistics & Doesn't handle & $\begin{array}{l}\text { Handles return, repair \& } \\
\text { disposal of failed components }\end{array}$ \\
\hline Performance matrix & Fill rate & Product availability (Uptime) \\
\hline $\begin{array}{l}\text { Inventory turn (the more the } \\
\text { batter ) }\end{array}$ & 6 to 50 a year & 1 to 4 a years \\
\hline
\end{tabular}

Creating service products (Cohen, Agrawal \& Agrawal, 2006):- The quickest way for companies to meet response targets is to replace the failed products with the whole end product unit that they have positioned on customers site. By contrast, the most economical way, to meet a service demand is to replace from the central facility with only the broken piece parts.

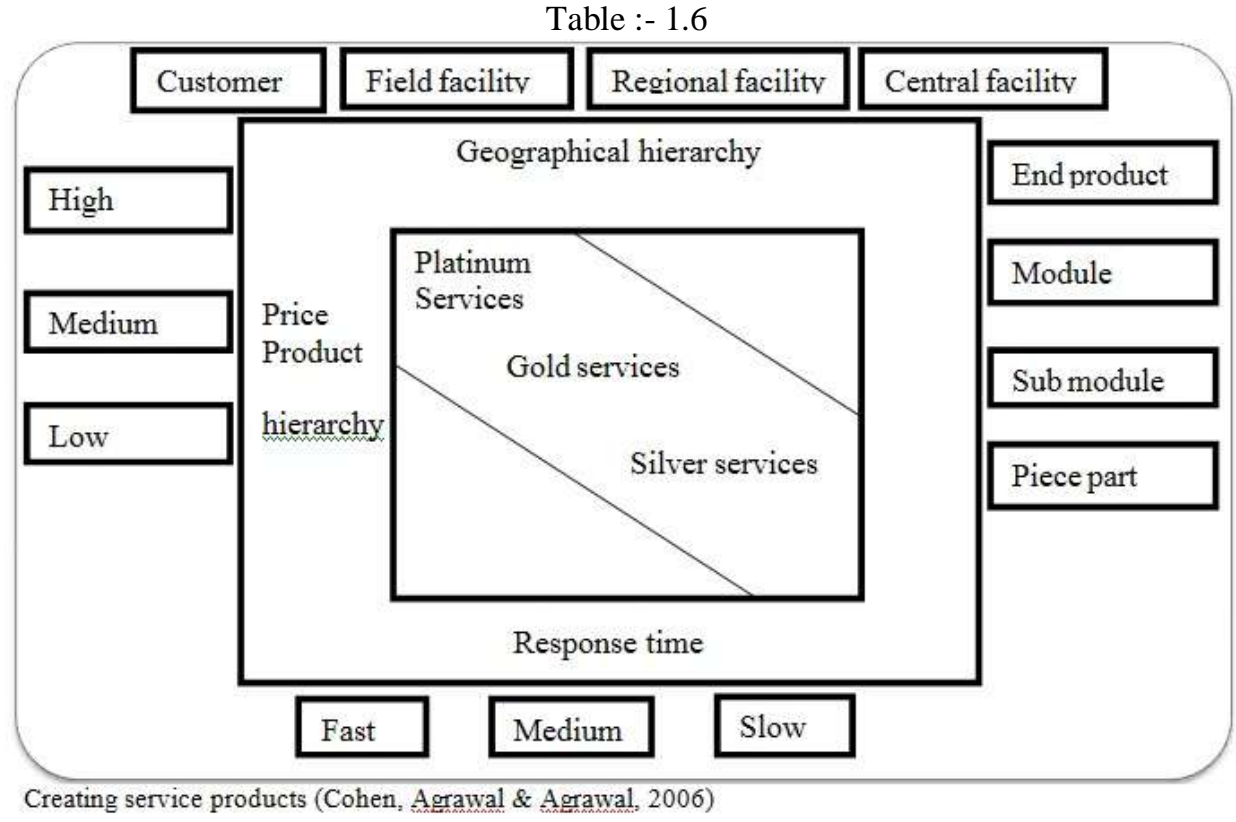

Two after sales service models: - "Who is responsible to take care of the after sales service? One is the manufacturer or its subordinate \& other is dealer of the manufacturer who is responsible for the service work. Problem statement:- 
Herbig \&Palumbo (1993) found that most obvious problem is the spare parts problem, which includes:-

1. Maintaining the expensive spare parts.

2. Incurring shipping \& importation delays in receiving the spare parts from some central storage.

If manufacturer let the dealers to take care of after sales service totally, there is the advantage that the storage way of spare parts might be more appropriate, because the dealers are more familiar with the conditions of the machines which they sold. They could have a good forecasting of spare parts inventory, moreover the dealers have more proximity to customers, and then their response for service might be quicker. However there is tradeoff between the service performance and service cost, and hence it should be well balanced.

Herbig \& Palumbo (1993) researched the after sales market in Japan \& in the United States and made two markets as the representation of Eastern \& the Western market. They found that, Japanese customers expect prompt service \& availability of the full line of parts for any major purchase. They expect after sales service is the part of purchase with longer warranty periods \& that is why in Japanese market after sales service managed directly by the manufacturer itself.

Another research result made by Wilson, Bostrom \& Lundin (1999), states that "East customers" want instant service \& they want it for free \& customers in "North America" are willing to pay in advance for the after sales service, in the form of service contract. Western customer seems to be more patient if they faced the problem such as machines failed as compared with the Eastern customers.

Wilson, Bostrom \& Lundin (1999) defined the after-sales service performing in five activities when it is handled through a distribution network, and these are:-

i. Installation

ii. $\quad$ Training

iii. Routine maintenance

iv. Emergency repair

v. Parts supply

vi. Software service

Wilson, Bostrom, \& Lundin (1999) found the similar results that pointed out, the Far East market has more difficult areas to service, both with regard to expectation of customer and communication problems.

After sales service is the prompt delivery of the benefits to the customers. In the after sales service, there are work of knowledge transferring, cost effective maintenance, repair and replacement. Moreover the good after sales service is caused by the factors of location, manufacturer, and dealer supporting \& service capacity.

\section{Research methodology:-}

The research design methodology used here is "Descriptive Research" which is also known as "Statistical Research". The main goal of this type of research is to describe the data and characteristics about what is being studied. The idea behind this type of research is to study frequencies, average, and other statistical calculations. Descriptive research is the exploration of the existing certain phenomena. Descriptive research is mainly done when a researcher wants to gain better understanding of a topic, in this case the rationale behind how to retain the customer, determine the voice of customers to implement it into the services being offered by BGGTS, \& steps BGGTS could take to further strengthen the relationship with their customers.

\section{$>\quad$ A)) Sampling techniques:-}

The sampling technique used is Non-probabilistic sampling method. Non-probabilistic sampling is a sampling technique where the sample selected for study does not involve random selection from the population. In our research since it was industry specific research, it was ideal to go for "Judgmental sampling" or "Purposive sampling" where the researcher chooses the sample based on who they think would be appropriate for the study. This is used primarily when there is limited number of people that have expertise in the area being researched. Only mechanical background employees who have expertise in "Gas Turbine" sectors were considered for study. Also the questionnaire was circulated to some BGGTS employees to check the consistency of the output across backgrounds characteristics.

Sample plan:-The sampling plan consists mainly of primary data collection. The following methods were used to gather information as part of primary data collection:-

\section{$>\quad$ B)) Focus group discussions:-}

BGGTS, IPGCL \& PPCL employees has been identified as focus groups and there inputs on how the system can be modified are taken into consideration and the questionnaire is modified accordingly. The end result of focus group discussion is a clearly defined objective for the questionnaire. Based on the focus group discussions, the factors that can effect decision making where selected as the variables of the questionnaire. A Well designed questionnaire whose objective is to classify the respondents according to the age group characteristics, study the rationale under which they make a choice of BGGTS for the services, were circulated in power sector companies. 
$>\quad$ C)) Designing questionnaire:-

The necessary questions and the appropriate scaling is identified and the information's to be collected were classified into 4 parts.

1. Background characteristics: - Nominal scale has been identified to gather all data regarding background characteristics.

2. Variable under study: - The variables that have been identified \& which could have an effect on the decision making in opting BGGTS for services to be delivered. Interval scale of 5 point scale (Likert Scale) has been identified to capture the output data regarding the variable under study.

3. Test parameters: - The various parameters that need to be tested on the Companies opting for BGGTS for the services being delivered. Interval scale or 5 point scale (Likert scale) has been identified to capture the output data regarding the parameters to be tested.

4. Other factors: - Factors that could affect the decision making on the basis of external factors such as competitive environment and technology enhancements has been identified \& Interval or rating scale has been identified to capture the output data regarding the other factors.

\section{$>\quad$ D)) Questionnaires validity test:-}

The questionnaires are subjected to content validity check using "Lawshe formulae" for content validity ratio (CVR).

It measures the essentiality of a question in a questionnaire.

Content validity ratio $=(2 \mathrm{Ne} / \mathrm{N})-1$

Where $\mathrm{Ne}=$ Number of panelists indicating that question is essential.

- $\quad \mathrm{N}=$ Total number of panelists

The number of panelists was selected to be $5 \&$ the questionnaires were circulated and the response for each item was noted. Items with CVR ratio $<0.5$ were dropped. The final questionnaire was then formulated.

Scale: ALL VARIABLES

\section{Reliability Test:-}

\begin{tabular}{|l|l|r|r|}
\hline \multicolumn{3}{|c|}{ Case Processing Summary } \\
\hline \multirow{3}{*}{ Cases } & \multicolumn{1}{|c|}{ N } & $\%$ \\
& Valid & 9 & 100.0 \\
\cline { 2 - 4 } & Excluded & & .0 \\
\cline { 2 - 4 } & Total & 0 & 100.0 \\
\hline a. Listwise deletion based on all variables in the procedure. & 9 & \\
\hline
\end{tabular}

Table:- 1.7

\begin{tabular}{|c|c|}
\hline \multicolumn{2}{|c|}{ Reliability Statistics } \\
\hline $\begin{array}{c}\text { Cronbach's } \\
\text { Alpha }\end{array}$ & N of Items \\
\hline .737 & \\
\hline
\end{tabular}

Inference:- Since the Cronbach's Alpha value is greater than $.6(.737>.6)$, hence data are reliable.

Data Analysis \& Interpretation: -

Outcome of frequency test being supported by Pie chart (Case processing summary)

\begin{tabular}{|c|c|c|c|c|c|}
\hline \multicolumn{6}{|c|}{ Company name } \\
\hline & & Frequency & Percent & Valid Percent & $\begin{array}{c}\text { Cumulative } \\
\text { Percent }\end{array}$ \\
\hline \multirow[t]{3}{*}{ Valid } & IPGCL & 3 & 33.3 & 33.3 & 33.3 \\
\hline & PPCL & 6 & 66.7 & 66.7 & 100.0 \\
\hline & Total & 9 & 100.0 & 100.0 & \\
\hline
\end{tabular}

Inference:- Majority of employee are from PPCL, with $66.7 \%$ as compared by IPGCL which has $33.3 \%$ of employees.

Sex 


\begin{tabular}{|l|r|r|r|r|}
\hline & Frequency & Percent & Valid Percent & $\begin{array}{c}\text { Cumulative } \\
\text { Percent }\end{array}$ \\
\hline Valid male & 9 & 100.0 & 100.0 & 100.0 \\
\hline
\end{tabular}

Inference:- All the participants are male only.

\begin{tabular}{|c|c|c|c|c|c|}
\hline \multicolumn{6}{|c|}{ Age } \\
\hline & & Frequency & Percent & Valid Percent & $\begin{array}{l}\text { Cumulative } \\
\text { Percent }\end{array}$ \\
\hline \multirow{4}{*}{ Valid } & $<30$ & 4 & 44.4 & 44.4 & 44.4 \\
\hline & $30-45$ & 2 & 22.2 & 22.2 & 66.7 \\
\hline & $>45$ & 3 & 33.3 & 33.3 & 100.0 \\
\hline & Total & 9 & 100.0 & 100.0 & \\
\hline
\end{tabular}

Inference:- Maximum number of participants are smaller than 30 years age group, with $44.4 \%$, followed by greater than 45 years age group, with $33.3 \%$ and least number of participants are from 30-45 years age group, with $22.2 \%$ of the total employees.

\begin{tabular}{|l|r|r|r|r|}
\hline & Service where 5>4>3>2>1 \\
\hline & Frequency & Percent & Valid Percent & $\begin{array}{c}\text { Cumulative } \\
\text { Percent }\end{array}$ \\
\hline Valid & 2 & 22.2 & 22.2 & 22.2 \\
& 2 & 22.2 & 22.2 & 44.4 \\
& 5 & 55.6 & 55.6 & 100.0 \\
5 & 9 & 100.0 & 100.0 & \\
\hline
\end{tabular}

Inference:- For service 5, which is the greatest possible rating has been rated by maximum employees, with $55.6 \%$,followed by $2 \& 3$ which is rated by equal number of employees, with $22.2 \%$ each of the total employees.

documentation where $5>4>3>2>1$

\begin{tabular}{|c|c|c|c|c|c|}
\hline & & Frequency & Percent & Valid Percent & $\begin{array}{c}\text { Cumulative } \\
\text { Percent }\end{array}$ \\
\hline \multirow[t]{3}{*}{ Valid } & 4 & 5 & 55.6 & 55.6 & 55.6 \\
\hline & 5 & 4 & 44.4 & 44.4 & 100.0 \\
\hline & Total & 9 & 100.0 & 100.0 & \\
\hline
\end{tabular}

Inference:- For documentation 4 , which is the $2^{\text {nd }}$ highest rating has been assigned by maximum number of employees, with $55.6 \%$, followed by 5 , which is the highest rating by $44.4 \%$ of the total employees.

\begin{tabular}{|c|c|c|c|c|c|}
\hline & & Frequency & Percent & Valid Percent & $\begin{array}{c}\text { Cumulative } \\
\text { Percent }\end{array}$ \\
\hline \multirow[t]{3}{*}{ Valid } & 4 & 3 & 33.3 & 33.3 & 33.3 \\
\hline & 5 & 6 & 66.7 & 66.7 & 100.0 \\
\hline & Total & 9 & 100.0 & 100.0 & \\
\hline
\end{tabular}

Inference:- For maintenance 5, which is the highest rating has been assigned by maximum number of employees, with $66.7 \%$,followed by 4 , which is the second highest rating by $33.3 \%$ of total employee. 
Research Study On After Sales Services of Gas Turbines online support where $5>4>3>2>1$

\begin{tabular}{|c|c|c|c|c|c|}
\hline & & Frequency & Percent & Valid Percent & $\begin{array}{c}\text { Cumulative } \\
\text { Percent }\end{array}$ \\
\hline \multirow[t]{4}{*}{ Valid } & 3 & 2 & 22.2 & 22.2 & 22.2 \\
\hline & 4 & 1 & 11.1 & 11.1 & 33.3 \\
\hline & 5 & 6 & 66.7 & 66.7 & 100.0 \\
\hline & Total & 9 & 100.0 & 100.0 & \\
\hline
\end{tabular}

Inference:- For online support 5, which is the highest rating point has been assigned by $66.7 \%$, followed by 3 , which is the third highest rating by $22.2 \%$, and the second highest rating 4 , has been assigned by least number of imployees with $11.1 \%$ of the total employees

parts supply where $5>4>3>2>1$

\begin{tabular}{|c|c|c|c|c|c|}
\hline & & Frequency & Percent & Valid Percent & $\begin{array}{c}\text { Cumulative } \\
\text { Percent }\end{array}$ \\
\hline \multirow[t]{3}{*}{ Valid } & 4 & 4 & 44.4 & 44.4 & 44.4 \\
\hline & 5 & 5 & 55.6 & 55.6 & 100.0 \\
\hline & Total & 9 & 100.0 & 100.0 & \\
\hline
\end{tabular}

Inference:- For parts supply 5, which is the highest rating has been assigned by maximum number of employees $55.6 \%$, followed by 4 , which is the second highest rating, by $44.4 \%$ of employees.

upgrades where $5>4>3>2>1$

\begin{tabular}{|c|c|c|c|c|c|}
\hline & & Frequency & Percent & Valid Percent & $\begin{array}{c}\text { Cumulative } \\
\text { Percent }\end{array}$ \\
\hline \multirow[t]{4}{*}{ Valid } & 3 & 3 & 33.3 & 33.3 & 33.3 \\
\hline & 4 & 3 & 33.3 & 33.3 & 66.7 \\
\hline & 5 & 3 & 33.3 & 33.3 & 100.0 \\
\hline & Total & 9 & 100.0 & 100.0 & \\
\hline
\end{tabular}

Infrence:- For upgrades 5 which is the highest,4 second highest \&,3 third highest ratings has been assigned equally by $33.3 \%$ of the total employees respectively.

high combined cycle efficiency

\begin{tabular}{|ll|r|r|r|r|}
\hline & Frequency & Percent & Valid Percent & \multicolumn{2}{c|}{$\begin{array}{c}\text { Cumulative } \\
\text { Percent }\end{array}$} \\
\hline Valid & strongly agree & 4 & 44.4 & 44.4 & 44.4 \\
& agree & 5 & 55.6 & 55.6 & 100.0 \\
& Total & 9 & 100.0 & 100.0 & \\
\hline
\end{tabular}

For high combined cycle efficiency 5 , which is the highest rating has been assigned by maximum number of employees, $55.6 \%$, followed by 4 which is the second highest rating by $44.4 \%$ of the total employees.

Low NOx emission

\begin{tabular}{|c|c|c|c|c|c|}
\hline & & Frequency & Percent & Valid Percent & $\begin{array}{c}\text { Cumulative } \\
\text { Percent }\end{array}$ \\
\hline \multirow[t]{3}{*}{ Valid } & strongly agree & 3 & 33.3 & 33.3 & 33.3 \\
\hline & agree & 6 & 66.7 & 66.7 & 100.0 \\
\hline & Total & 9 & 100.0 & 100.0 & \\
\hline
\end{tabular}


Research Study On After Sales Services of Gas Turbines

Inference:- For Low NOx emission maximum number of people are agree 66.7, followed by strongly agree, which is supported by 33.3

low specific cost (per KW)

\begin{tabular}{|ll|r|r|r|r|}
\hline & & & & \multicolumn{2}{c|}{$\begin{array}{c}\text { Cumulative } \\
\text { Percent }\end{array}$} \\
\hline Valid & Strongly agree & 6 & 66.7 & 66.7 & 66.7 \\
& Agree & 3 & 33.3 & 33.3 & 100.0 \\
& 9 & 100.0 & 100.0 & \\
\hline
\end{tabular}

Infrance:- For low specific cost maximum number of employees has prefered Strongly agree $66.7 \%$, followed by $33.3 \%$ are agree.

High availability

\begin{tabular}{|ll|r|r|r|r|}
\hline & & & & \multicolumn{2}{c|}{$\begin{array}{c}\text { Cumulative } \\
\text { Percent }\end{array}$} \\
\hline Valid & Strongly agree & 6 & 66.7 & 66.7 & 66.7 \\
& Agree & 3 & 33.3 & 33.3 & 100.0 \\
& Total & 9 & 100.0 & 100.0 & \\
\hline
\end{tabular}

Inference:- For high availability maximum number of employees referred Strongly agree $66.7 \%$, followed by $33.3 \%$ for agree.

\begin{tabular}{|ll|r|r|r|r|}
\hline & & & & Cumulative \\
& & Frequency & Percent & Valid Percent & \multicolumn{1}{c|}{ Percent } \\
\hline Valid & Strongly agree & 4 & 44.4 & 44.4 & 44.4 \\
& Agree & 5 & 55.6 & 55.6 & 100.0 \\
& Total & 9 & 100.0 & 100.0 & \\
\hline
\end{tabular}

Inference:- For operating flexibility maximum number of employees referred for Agree $55.6 \%$, followed by $44.4 \%$ for strongly agree.

High reliability

\begin{tabular}{|ll|r|r|r|r|}
\hline & & & & \multicolumn{2}{c|}{$\begin{array}{c}\text { Cumulative } \\
\text { Percent }\end{array}$} \\
\hline Valid & Strongly agree & 5 & 55.6 & 55.6 & 55.6 \\
& Agree & 4 & 44.4 & 44.4 & 100.0 \\
& Total & 9 & 100.0 & 100.0 & \\
\hline
\end{tabular}

Inference:- For high reliability maximum number of people referred for Strongly agree $55.6 \%$, followed by $44.4 \%$ for agree.

\begin{tabular}{|ll|r|r|r|r|}
\hline & & & \\
& & High simple cycle efficiency & \multicolumn{2}{c|}{$\begin{array}{c}\text { Cumulative } \\
\text { Percent }\end{array}$} \\
\hline Valid & strongly agree & 6 & 66.7 & 66.7 & 66.7 \\
& Agree & 3 & 33.3 & 33.3 & 100.0 \\
& Total & 9 & 100.0 & 100.0 & \\
\hline
\end{tabular}

Inference:- For High simple cycle efficiency maximum number of employees referred for strongly agree $66.7 \%$, followed by $33.3 \%$ for agree

\section{Cooling Technology}


Research Study On After Sales Services of Gas Turbines

\begin{tabular}{|ll|r|r|r|r|}
\hline & & & & \multicolumn{2}{c|}{$\begin{array}{c}\text { Cumulative } \\
\text { Percent }\end{array}$} \\
\hline Valid & Strongly agree & 4 & 44.4 & 44.4 & 44.4 \\
& Agree & 5 & 55.6 & 55.6 & 100.0 \\
& Total & 9 & 100.0 & 100.0 & \\
\hline
\end{tabular}

Inference:- For cooling technology maximum number of employees reffered for Agree $55.5 \%$, followed by $44.4 \%$ for strongly agree

Firing temperature

\begin{tabular}{|c|c|c|c|c|c|}
\hline & & Frequency & Percent & Valid Percent & $\begin{array}{l}\text { Cumulative } \\
\text { Percent }\end{array}$ \\
\hline \multirow[t]{3}{*}{ Valid } & Strongly agree & 4 & 44.4 & 44.4 & 44.4 \\
\hline & Agree & 5 & 55.6 & 55.6 & 100.0 \\
\hline & Total & 9 & 100.0 & 100.0 & \\
\hline
\end{tabular}

Inference:- For Firing temperature maximum number of employees referred for Agree 55.6\%, followed by 44.4 $\%$ for strongly agree.

\section{Pressure ratio}

\begin{tabular}{|c|c|c|c|c|c|}
\hline & & Frequency & Percent & Valid Percent & $\begin{array}{c}\text { Cumulative } \\
\text { Percent }\end{array}$ \\
\hline \multirow[t]{3}{*}{ Valid } & Strongly agree & 6 & 66.7 & 66.7 & 66.7 \\
\hline & Agree & 3 & 33.3 & 33.3 & 100.0 \\
\hline & Total & 9 & 100.0 & 100.0 & \\
\hline
\end{tabular}

Inference:- For Pressure ratio maximum number of employees referred for strongly agree $66.7 \%$, followed by $33.3 \%$ for agree.

\begin{tabular}{|ll|r|r|r|r|}
\hline \multicolumn{1}{|c|}{ Mass flow } \\
\hline & Frequency & Percent & Valid Percent & $\begin{array}{c}\text { Cumulative } \\
\text { Percent }\end{array}$ \\
\hline Valid & strongly agree & 33.3 & 33.3 & 33.3 \\
& agree & 5 & 55.6 & 55.6 & 88.9 \\
neutral & 1 & 11.1 & 11.1 & 100.0 \\
Total & 9 & 100.0 & 100.0 & \\
\hline
\end{tabular}

Inference:- For Mass flow maximum number of employees referred for agree $55.6 \%$, followed by $33.3 \%$ for Strongly agree where as $11.1 \%$ employees reffered for neutral.

Planned outage frequency

\begin{tabular}{|ll|r|r|r|r|}
\hline & Frequency & Percent & Valid Percent & \multicolumn{2}{c|}{$\begin{array}{c}\text { Pumulative } \\
\text { Percent }\end{array}$} \\
\hline Valid & important & 3 & 33.3 & 33.3 & 33.3 \\
& Quite important & 3 & 33.3 & 33.3 & 66.7 \\
& Very important & 3 & 33.3 & 33.3 & 100.0 \\
& Total & 9 & 100.0 & 100.0 & \\
\hline
\end{tabular}


Research Study On After Sales Services of Gas Turbines

Inference:- For Planned outage frequency all employees reffered equally for important, quite important, \& very important $33.3 \%$ respectively.

utage duration

\begin{tabular}{|ll|r|r|r|r|}
\hline & & & & \multicolumn{2}{c|}{$\begin{array}{c}\text { Cumulative } \\
\text { Percent }\end{array}$} \\
\hline Valid & Quite important & 3 & 33.3 & 33.3 & 33.3 \\
& Very important & 6 & 66.7 & 66.7 & 100.0 \\
& Total & 9 & 100.0 & 100.0 & \\
\hline
\end{tabular}

Inference:-For outage duration maximum number of employees reffered Very important $66.7 \%$, followed by $33.3 \%$ for Quite important.

\begin{tabular}{|ll|r|r|r|r|}
\hline & & & \\
& & Frequency & Percent & Valid Percent & \multicolumn{2}{c|}{$\begin{array}{c}\text { Pumulative } \\
\text { Percent }\end{array}$} \\
\hline Valid & Important & 2 & 22.2 & 22.2 & 22.2 \\
& Quite important & 4 & 44.4 & 44.4 & 66.7 \\
& Very important & 3 & 33.3 & 33.3 & 100.0 \\
& Total & 9 & 100.0 & 100.0 & \\
\hline
\end{tabular}

Inference:- For parts replacement frequency maximum number of employees reffered for Quite important 44.4 $\%$, followed by very important $33.33 \%$, and least number of employees reffered for important $22.2 \%$.

Parts replacement costs

\begin{tabular}{|ll|r|r|r|r|}
\hline & & & & Cumulative \\
& & Frequency & Percent & Valid Percent & \multicolumn{2}{|c|}{\begin{tabular}{c} 
Percent \\
\hline Valid
\end{tabular} Quite important } & 3 & 33.3 & 33.3 & 33.3 \\
& Very important & 6 & 66.7 & 66.7 & 100.0 \\
& Total & 9 & 100.0 & 100.0 & \\
\hline
\end{tabular}

Inference:- For parts replacement costs maximum number of employees referred for very important $66.7 \%$, followed by $33.3 \%$ for quite important.

Uncertainty repair/maintenance

\begin{tabular}{|ll|r|r|r|r|}
\hline & Frequency & Percent & Valid Percent & $\begin{array}{c}\text { Cumulative } \\
\text { Percent }\end{array}$ \\
\hline Valid & Quite important & 2 & 22.2 & 22.2 & 22.2 \\
& Very important & 7 & 77.8 & 77.8 & 100.0 \\
Total & 9 & 100.0 & 100.0 & \\
\hline
\end{tabular}

Inference:- For parts Uncertainty repair/maintenance maximum number of employees referred for very important $77.8 \%$, followed by $22.2 \%$ for quite important.

daily operation costs

\begin{tabular}{|ll|r|r|r|r|}
\hline & & & & \multicolumn{2}{c|}{$\begin{array}{c}\text { Cumulative } \\
\text { Percent }\end{array}$} \\
\hline Valid & Important & 2 & 22.2 & 22.2 & 22.2 \\
& Quite important & 1 & 11.1 & 11.1 & 33.3 \\
& Very important & 6 & 66.7 & 66.7 & 100.0
\end{tabular}


Research Study On After Sales Services of Gas Turbines

\begin{tabular}{|rl|r|r|r|r|}
\hline \multicolumn{7}{|c|}{ daily operation costs } \\
\hline & & Frequency & Percent & Valid Percent & $\begin{array}{c}\text { Cumulative } \\
\text { Percent }\end{array}$ \\
\hline Valid & Important & 2 & 22.2 & 22.2 & 22.2 \\
& Quite important & 1 & 11.1 & 11.1 & 33.3 \\
& Very important & 6 & 66.7 & 66.7 & 100.0 \\
Total & 9 & 100.0 & 100.0 & \\
\hline
\end{tabular}

Inference:- For Daily operation costs maximum number of employees referred for very important $66.7 \%$, followed by $22.2 \%$ for important, and only $11.1 \%$ reffered for quite important.

\begin{tabular}{|c|c|c|c|c|c|}
\hline \multicolumn{6}{|c|}{ NOx abatement } \\
\hline & & Frequency & Percent & Valid Percent & $\begin{array}{c}\text { Cumulative } \\
\text { Percent }\end{array}$ \\
\hline Valid & important & 1 & 11.1 & 11.1 & 11.1 \\
\hline & quite important & 4 & 44.4 & 44.4 & 55.6 \\
\hline & very important & 4 & 44.4 & 44.4 & 100.0 \\
\hline & Total & 9 & 100.0 & 100.0 & \\
\hline
\end{tabular}

Inference:- For NOx abatement equal number of employees referred for quite important \& very important 44.4 $\%$ respectively, followed by $11.1 \%$ for important.

\begin{tabular}{|ll|r|r|r|r|}
\hline & Long time surety of price \\
\hline & Frequency & Percent & Valid Percent & $\begin{array}{c}\text { Cumulative } \\
\text { Percent }\end{array}$ \\
\hline Valid & Strongly agree & 7 & 77.8 & 77.8 & 77.8 \\
& Agree & 1 & 11.1 & 11.1 & 88.9 \\
Neutral & 1 & 11.1 & 11.1 & 100.0 \\
Total & 9 & 100.0 & 100.0 & \\
\hline
\end{tabular}

Inference:- For Long time surety of price maximum number of employees referred for strongly agree $77.8 \%$, followed by $11.1 \%$ equally for both Agree and neutral respectively.

\section{Provision of high quality outage services}

\begin{tabular}{|ll|r|r|r|r|}
\hline & & & & Cumulative \\
& & Frequency & Percent & Valid Percent & \multicolumn{2}{c|}{ Percent } \\
\hline Valid & Strongly agree & 7 & 77.8 & 77.8 & 77.8 \\
& 1 & 11.1 & 11.1 & 88.9 \\
& Agree & 1 & 11.1 & 11.1 & 100.0 \\
Neutral & 9 & 100.0 & 100.0 & \\
Total & 9 & \\
\hline
\end{tabular}

Inference:- For Provision of high quality outage services maximum number of employees referred for Strongly agree $77.8 \%$, followed equally by $11.1 \%$ for both agree \& neutral respectively.

Local project management \& execution

\begin{tabular}{|ll|r|r|r|r|}
\hline & & & & \multicolumn{2}{c|}{$\begin{array}{c}\text { Cumulative } \\
\text { Percent }\end{array}$} \\
\hline Valid & Strongly agree & 5 & 55.6 & 55.6 & 55.6 \\
& Agree & 4 & 44.4 & 44.4 & 100.0 \\
& Total & 9 & 100.0 & 100.0 & \\
\hline
\end{tabular}


Inference:- For Local project management \& execution maximum number of employees referred for strongly agree $55.6 \%$, followed by $44.4 \%$ for agree.

Rapid supply of key parts in case of emergency failures

\begin{tabular}{|l|r|r|r|r|}
\hline & Frequency & Percent & Valid Percent & $\begin{array}{c}\text { Cumulative } \\
\text { Percent }\end{array}$ \\
\hline Valid Strongly agree & 9 & 100.0 & 100.0 & 100.0 \\
\hline
\end{tabular}

Inference:- For rapid supply of key parts in case of emergency failures all employees opted only for strongly agree $100 \%$.

Remote expert monitoring of plant

\begin{tabular}{|ll|r|r|r|r|}
\hline & & & & Cumulative \\
& Frequency & Percent & Valid Percent & \multicolumn{2}{|c|}{ Percent } \\
\hline Valid & Strongly agree & 1 & 11.1 & 11.1 & 11.1 \\
& Agree & 4 & 44.4 & 44.4 & 55.6 \\
& Neutral & 4 & 44.4 & 44.4 & 100.0 \\
Total & 9 & 100.0 & 100.0 & \\
\hline
\end{tabular}

Inference:- For Remote expert monitoring of plant maximum number of employees referred equallyfor agree \& neutral $44.47 \%$ respectively, followed by $11.1 \%$ for strongly agree.

An on site expert to provide immediate advice \&coordinate activities

\begin{tabular}{|ll|r|r|r|r|}
\hline & & & & Cumulative \\
& & Frequency & Percent & Valid Percent & \multicolumn{1}{c|}{ Percent } \\
\hline Valid & strongly agree & 3 & 33.3 & 33.3 & 33.3 \\
& agree & 5 & 55.6 & 55.6 & 88.9 \\
& 1 & 11.1 & 11.1 & 100.0 \\
neutral & 9 & 100.0 & 100.0 & \\
\hline
\end{tabular}

Inference:- For An on site expert to provide immediate advice \& cooreinate activities maximum number of employees referred for agree $55.6 \%$, followed by $33.3 \%$ for strongly agree, and $11.1 \%$ for neutral

Fuel system conversions

\begin{tabular}{|ll|r|r|r|r|}
\hline & & & & Cumulative \\
& & Frequency & Percent & Valid Percent & \multicolumn{2}{|c|}{ Percent } \\
\hline Valid & Strongly agree & 5 & 55.6 & 55.6 & 55.6 \\
& Agree & 1 & 11.1 & 11.1 & 66.7 \\
& Neutral & 1 & 11.1 & 11.1 & 77.8 \\
Disagree & 2 & 22.2 & 22.2 & 100.0 \\
Total & 9 & 100.0 & 100.0 & \\
\hline
\end{tabular}

Inference:- For Fuel system conversions maximum number of employees referred for strongly agree 55.6\%, followed by $22.2 \%$ for disagree, and followed by $11.1 \%$ for both agree and neutral respectively.

Emission abatement systems

\begin{tabular}{|ll|r|r|r|r|}
\hline & Frequency & Percent & Valid Percent & \multicolumn{2}{|c|}{ Pumulative } \\
\hline Valid & Strongly agree & 4 & 44.4 & 44.4 & 44.4 \\
& Agree & 2 & 22.2 & 22.2 & 66.7 \\
& Neutral & 2 & 22.2 & 22.2 & 88.9 \\
Disagree & 1 & 11.1 & 11.1 & 100.0
\end{tabular}


Research Study On After Sales Services of Gas Turbines

\begin{tabular}{|ll|r|r|r|r|}
\hline & Emission abatement systems \\
\hline & Frequency & Percent & Valid Percent & \multicolumn{2}{c|}{$\begin{array}{c}\text { Cumulative } \\
\text { Percent }\end{array}$} \\
\hline Valid & Strongly agree & 4 & 44.4 & 44.4 & 44.4 \\
& 2 & 22.2 & 22.2 & 66.7 \\
Agree & 2 & 22.2 & 22.2 & 88.9 \\
Neutral & 1 & 11.1 & 11.1 & 100.0 \\
Disagree & 9 & 100.0 & 100.0 & \\
Total & & & \\
\hline
\end{tabular}

Inference:- For Emission abatement systems maximum number of employees referred for strongly agree 44.4\%, followed by $22.2 \%$ for both agree and neutral respectively, and followed by $11.1 \%$ for disagree and neutral respectively.

Auxiliary system modification

\begin{tabular}{|ll|r|r|r|r|}
\hline & & & & \multicolumn{2}{c|}{$\begin{array}{c}\text { Cumulative } \\
\text { Percent }\end{array}$} \\
\hline Valid & strongly agree & 4 & 44.4 & 44.4 & 44.4 \\
& Agree & 3 & 33.3 & 33.3 & 77.8 \\
& Neutral & 2 & 22.2 & 22.2 & 100.0 \\
& Total & 9 & 100.0 & 100.0 & \\
\hline
\end{tabular}

Inference:- For Auxiliary system modification maximum number of employees referred for strongly agree $44.4 \%$, followed by $23.3 \%$ for agree, and followed by $2.2 \%$ for neutral.

Instrumentation \& control system modification

\begin{tabular}{|ll|r|r|r|r|}
\hline & & & & Cumulative \\
& & Frequency & Percent & Valid Percent & \multicolumn{1}{c|}{ Percent } \\
\hline Valid & Strongly agree & 5 & 55.6 & 55.6 & 55.6 \\
& Agree & 2 & 22.2 & 22.2 & 77.8 \\
& Neutral & 2 & 22.2 & 22.2 & 100.0 \\
Total & 9 & 100.0 & 100.0 & \\
\hline
\end{tabular}

Inference:- For Instrumentation \& control system modification maximum number of employees referred for strongly agree $55.6 \%$, followed by $22.2 \%$ for both agree and neutral respectively.

Corrosion monitoring

\begin{tabular}{|ll|r|r|r|r|}
\hline & & & & Cumulative \\
& & Frequency & Percent & Valid Percent & \multicolumn{2}{|c|}{ Percent } \\
\hline Valid & strongly agree & 6 & 66.7 & 66.7 & 66.7 \\
& 2 & 22.2 & 22.2 & 88.9 \\
& agree & 1 & 11.1 & 11.1 & 100.0 \\
& disagree & 9 & 100.0 & 100.0 & \\
Total & & & \\
\hline
\end{tabular}

Inference:- For Corrosion monitoring maximum number of employees referred for strongly agree $66.7 \%$, followed by $22.2 \%$ for agree and $11.1 \%$ for disagree. 


\begin{tabular}{|ll|r|r|r|r|}
\hline \multicolumn{5}{|c|}{ Air sampling } \\
& & Frequency & Percent & Valid Percent & $\begin{array}{c}\text { Cumulative } \\
\text { Percent }\end{array}$ \\
\hline Valid & Strongly agree & 4 & 44.4 & 44.4 & 44.4 \\
& Agree & 3 & 33.3 & 33.3 & 77.8 \\
Neutral & 1 & 11.1 & 11.1 & 88.9 \\
Disagree & 1 & 11.1 & 11.1 & 100.0 \\
Total & 9 & 100.0 & 100.0 & \\
\hline
\end{tabular}

Inference:- For Air sampling maximum number of employees referred for strongly agree $44.4 \%$, followed by $33.3 \%$ for agree, and $11.1 \%$ For both neutral and diagree respectively

\section{Material testing}

\begin{tabular}{|ll|r|r|r|r|}
\hline & & & & Cumulative \\
& Frequency & Percent & Valid Percent & \multicolumn{2}{|c|}{ Percent } \\
\hline Valid & Strongly agree & 5 & 55.6 & 55.6 & 55.6 \\
& 2 & 22.2 & 22.2 & 77.8 \\
Agree & 1 & 11.1 & 11.1 & 88.9 \\
Neutral & 1 & 11.1 & 11.1 & 100.0 \\
Disagree & 9 & 100.0 & 100.0 & \\
Total & &
\end{tabular}

Inference:- For Material testing maximum number of employees referred for strongly agree $55.6 \%$, followed by $22.2 \%$ for agree and $11.1 \%$ for both neutral and disagree respectively.

\begin{tabular}{|ll|r|r|r|r|}
\hline \multicolumn{7}{|c|}{ Site surveys } \\
& Frequency & Percent & Valid Percent & $\begin{array}{c}\text { Cumulative } \\
\text { Percent }\end{array}$ \\
\hline Valid & strongly agree & 2 & 22.2 & 22.2 & 22.2 \\
& agree & 5 & 55.6 & 55.6 & 77.8 \\
& 1 & 11.1 & 11.1 & 88.9 \\
neutral & 1 & 11.1 & 11.1 & 100.0 \\
disagree & 9 & 100.0 & 100.0 & \\
Total & & & \\
\hline
\end{tabular}

Inference:- For Site surveys maximum number of employees referred for agree 55.6\%, followed by $22.2 \%$ for strongly agree and $11.1 \%$ both for neutral and disagree respectively.

\begin{tabular}{|ll|r|r|r|r|}
\hline & Technical advisory services \\
\hline & Frequency & Percent & Valid Percent & $\begin{array}{c}\text { Cumulative } \\
\text { Percent }\end{array}$ \\
\hline Valid & Strongly agree & 5 & 55.6 & 55.6 & 55.6 \\
& Agree & 3 & 33.3 & 33.3 & 88.9 \\
& Strongly disagree & 1 & 11.1 & 11.1 & 100.0 \\
& Total & 9 & 100.0 & 100.0 & \\
\hline
\end{tabular}

Inference:- For Technical advisory services maximum number of employees referred for strongly agree $55.6 \%$, followed by $33.3 \%$ for agree and $11.1 \%$ for strongly disagree. 
Research Study On After Sales Services of Gas Turbines

\begin{tabular}{|ll|r|r|r|r|}
\hline \multicolumn{6}{|c|}{ services likes the most } \\
\hline & Frequency & Percent & Valid Percent & $\begin{array}{c}\text { Cumulative } \\
\text { Percent }\end{array}$ \\
\hline Valid & OEM credentials & 3 & 33.3 & 33.3 & 33.3 \\
& design \& looks & 1 & 11.1 & 11.1 & 44.4 \\
quick service response time & 5 & 55.6 & 55.6 & 100.0 \\
Total & 9 & 100.0 & 100.0 & \\
\hline
\end{tabular}

Inference:- For Services likes the most maximum number of employees referred for Quick service response time $55.6 \%$, followed by $33.3 \%$ for OEM credentials and $11.1 \%$ for design and looks.

Preferred decision making factors

\begin{tabular}{|ll|r|r|r|r|}
\hline & Frequency & Percent & Valid Percent & $\begin{array}{c}\text { Cumulative } \\
\text { Percent }\end{array}$ \\
\hline Valid & Maintenance price & 1 & 11.1 & 11.1 & 11.1 \\
& 2 & 22.2 & 22.2 & 33.3 \\
Quick response & 3 & 33.3 & 33.3 & 66.7 \\
Knowledgeable \& polite & 3 & 33.3 & 33.3 & 100.0 \\
sales staff & 3 & 100.0 & 100.0 & \\
Manner of the personal & 9 & 100.0 & \\
Total & &
\end{tabular}

Inference:- For Preferred decision making factors maximum number of employees referred for both knowledgeable and polite sales staff $33.3 \%$ respectively,followed by $22.2 \%$ for quick responce and $11.1 \%$ for maintenance price.

Your next order will be to BGGTS

\begin{tabular}{|c|c|c|c|c|c|}
\hline & & Frequency & Percent & Valid Percent & $\begin{array}{c}\text { Cumulative } \\
\text { Percent }\end{array}$ \\
\hline \multirow[t]{4}{*}{ Valid } & strongly agree & 3 & 33.3 & 33.3 & 33.3 \\
\hline & agree & 3 & 33.3 & 33.3 & 66.7 \\
\hline & neutral & 3 & 33.3 & 33.3 & 100.0 \\
\hline & Total & 9 & 100.0 & 100.0 & \\
\hline
\end{tabular}

Inference:- For Your next order will be to BGGTS all employees referred equally for strongly agree, agree and neutral $33.3 \%$ respectively.

Satisfied by services delivered

\begin{tabular}{|ll|r|r|r|r|}
\hline & Frequency & Percent & Valid Percent & \multicolumn{2}{c|}{$\begin{array}{c}\text { Pumulative } \\
\text { Percent }\end{array}$} \\
\hline Valid & Strongly agree & 2 & 22.2 & 22.2 & 22.2 \\
& Agree & 5 & 55.6 & 55.6 & 77.8 \\
& 2 & 22.2 & 22.2 & 100.0 \\
Neutral & 9 & 100.0 & 100.0 & \\
Total & 9 & & \\
\hline
\end{tabular}

Inference:- For Satisfied by services delivered maximum number of employees referred for agree 55.6\%, followed by $22.2 \%$ for both strongly agree and neutral respectively. 
Research Study On After Sales Services of Gas Turbines

\begin{tabular}{|c|c|c|c|c|c|}
\hline \multicolumn{6}{|c|}{ Services worth money paid } \\
\hline & & Frequency & Percent & Valid Percent & $\begin{array}{c}\text { Cumulative } \\
\text { Percent }\end{array}$ \\
\hline \multirow[t]{4}{*}{ Valid } & Strongly agree & 6 & 66.7 & 66.7 & 66.7 \\
\hline & Agree & 2 & 22.2 & 22.2 & 88.9 \\
\hline & Neutral & 1 & 11.1 & 11.1 & 100.0 \\
\hline & Total & 9 & 100.0 & 100.0 & \\
\hline
\end{tabular}

Inference:- For Services worth money paid maximum number of employees referred for strongly agree $66.7 \%$, followed by $22.2 \%$ for agree and $11.1 \%$ neutral.

\begin{tabular}{|c|c|c|c|c|c|}
\hline \multicolumn{6}{|c|}{ BGGTS response time is satisfactory } \\
\hline & & Frequency & Percent & Valid Percent & $\begin{array}{c}\text { Cumulative } \\
\text { Percent }\end{array}$ \\
\hline \multirow[t]{4}{*}{ Valid } & Strongly agree & 6 & 66.7 & 66.7 & 66.7 \\
\hline & Agree & 2 & 22.2 & 22.2 & 88.9 \\
\hline & Disagree & 1 & 11.1 & 11.1 & 100.0 \\
\hline & Total & 9 & 100.0 & 100.0 & \\
\hline
\end{tabular}

Inference:- For BGGTS response time is satisfactory maximum number of employees referred for strongly agree $66.6 \%$, followed by $22.2 \%$ for agree and $11.1 \%$ disagree.

\begin{tabular}{|rl|r|r|r|r|}
\hline \multicolumn{7}{|c|}{ Technical support is satisfactory } \\
& Frequency & Percent & Valid Percent & $\begin{array}{c}\text { Cumulative } \\
\text { Percent }\end{array}$ \\
\hline Valid & Strongly agree & 4 & 44.4 & 44.4 & 44.4 \\
& Agree & 1 & 11.1 & 11.1 & 55.6 \\
Neutral & 4 & 44.4 & 44.4 & 100.0 \\
Total & 9 & 100.0 & 100.0 & \\
\hline
\end{tabular}

Inference:- For Technical support is satisfactory maximum number of employees referred equally bothfor strongly agree and neutral respectively, followed by $11.1 \%$ for agree.

Test for correlation:-

Table :- 1.8

Correlations

\begin{tabular}{|ll|r|r|}
\hline & & Age & $\begin{array}{c}\text { service where } \\
5>4>3>2>1\end{array}$ \\
\hline Age & Pearson Correlation & 1 & $.830^{* * *}$ \\
& Sig. (2-tailed) & .006 \\
& $\mathrm{~N}$ & 9 & 9 \\
\hline service where 5>4>3>2>1 & Pearson Correlation & $.830^{* * *}$ & 1 \\
& Sig. (2-tailed) & .006 & 9 \\
& $\mathrm{~N}$ & 9 & 9 \\
\hline
\end{tabular}

**. Correlation is significant at the 0.01 level (2-tailed).

Conclusion:- There is Strong positive correlation between Age and Services where $5>4>3>2>1$. There is significant difference.

Table :- 1.9 
ANOVA

\begin{tabular}{|l|r|r|r|r|r|}
\cline { 2 - 6 } \multicolumn{1}{l|}{ Age } & & & & \\
\hline Between Groups & Sum of Squares & df & Mean Square & F & Sig. \\
Within Groups & 5.689 & 2 & 2.844 & 14.222 & .005 \\
Total & 1.200 & 6 & .200 & & \\
\hline
\end{tabular}

Conclusion:- There is significant difference among the perception in the age group, <30, 30-45 \& >45, respondents in their choice of "Service" as the critical parameter for after sales service for their plant

\begin{tabular}{|c|c|c|c|}
\hline \multicolumn{4}{|c|}{ Correlations } \\
\hline & & Age & $\begin{array}{c}\text { documentation } \\
\text { where } \\
5>4>3>2>1\end{array}$ \\
\hline \multirow[t]{3}{*}{ Age } & Pearson Correlation & 1 & .625 \\
\hline & Sig. (2-tailed) & & .072 \\
\hline & $\mathrm{N}$ & 9 & 9 \\
\hline \multirow{3}{*}{$\begin{array}{l}\text { documentation where } \\
5>4>3>2>1\end{array}$} & Pearson Correlation & .625 & 1 \\
\hline & Sig. (2-tailed) & .072 & \\
\hline & $\mathrm{N}$ & 9 & 9 \\
\hline
\end{tabular}

Conclusion:- There is strong positive correlation between Age and Documentation where $5>4>3>2>1$. There is no significant difference

Table:- 2

Correlations

\begin{tabular}{|c|c|c|c|}
\hline & & Age & $\begin{array}{c}\text { maintenance } \\
\text { where } \\
5>4>3>2>1\end{array}$ \\
\hline \multirow[t]{3}{*}{ Age } & Pearson Correlation & 1 & $.718^{*}$ \\
\hline & Sig. (2-tailed) & & .029 \\
\hline & $\mathrm{N}$ & 9 & 9 \\
\hline \multirow{3}{*}{$\begin{array}{l}\text { maintenance where } \\
5>4>3>2>1\end{array}$} & Pearson Correlation & $.718^{*}$ & 1 \\
\hline & Sig. (2-tailed) & .029 & \\
\hline & $\mathrm{N}$ & 9 & 9 \\
\hline
\end{tabular}

*. Correlation is significant at the 0.05 level (2-tailed).

There is strong positive correlation between Age and Maintenance where 5>4>3>2>1

There is significant difference.

Table:- 2.1

\begin{tabular}{|c|c|c|c|c|c|}
\hline \multicolumn{6}{|c|}{ ANOVA } \\
\hline Age & & & & & \\
\hline & Sum of Squares & $\mathrm{df}$ & Mean Square & $\mathrm{F}$ & Sig. \\
\hline Between Groups & 3.556 & 1 & 3.556 & 7.467 & .029 \\
\hline Within Groups & 3.333 & 7 & .476 & & \\
\hline Total & 6.889 & 8 & & & \\
\hline
\end{tabular}

Conclusion:- There is significant difference among the perception in the age group, <30, 30-45 \& >45, respondents in their choice of "Maintenance" as the critical parameter for after sales service for their plant.

Table:- 2.2 


\begin{tabular}{|ll|r|r|}
\hline & & $\begin{array}{c}\text { online support } \\
\text { where } \\
5>4>3>2>1\end{array}$ \\
\hline Age & Pearson Correlation & 1 & .679 \\
& Sig. (2-tailed) & 9 & .044 \\
& $\mathrm{~N}$ & 9 & 9 \\
\hline online support where & Pearson Correlation & $.679^{*}$ & 1 \\
$5>4>3>2>1$ & Sig. (2-tailed) & .044 & 9 \\
& $\mathrm{~N}$ & 9 & 9 \\
\hline
\end{tabular}

*. Correlation is significant at the 0.05 level (2-tailed).

There is strong positive correlation between Age and Online support where $5>4>3>2>1$

There is significant difference.

Table :- 2.3

ANOVA

\begin{tabular}{|l|r|r|r|r|r|}
\cline { 2 - 6 } \multicolumn{1}{c|}{ Age } & & & & & \\
\hline & Sum of Squares & df & Mean Square & F & Sig. \\
\hline Between Groups & 3.556 & 2 & 1.778 & 3.200 & .113 \\
Within Groups & 3.333 & 6 & .556 & & \\
Total & 6.889 & 8 & & & \\
\hline
\end{tabular}

Conclusion:- There is no significant difference among the perception in the age group, $<30,30-45 \&>45$, respondents in their choice of "Online support" as the critical parameter for after sales service for their plant

\begin{tabular}{|ll|r|r|}
\hline \multicolumn{1}{|c|}{ Correlations } & & $\begin{array}{c}\text { parts supply } \\
\text { where } \\
5>4>3>2>1\end{array}$ \\
\hline Age & Age & 1 & .653 \\
& Pearson Correlation & & .056 \\
& Sig. (2-tailed) & 9 & 9 \\
\hline parts supply where & Pearson Correlation & .653 & 1 \\
$5>4>3>2>1$ & Sig. (2-tailed) & .056 & 9 \\
& N & 9 & 9 \\
\hline
\end{tabular}

Conclusion:-There is strong positive correlation between Age and parts supply where $5>4>3>2>1$. There is no significant difference.

\begin{tabular}{|ll|r|r|}
\hline & \multicolumn{1}{c|}{ Correlations } & $\begin{array}{c}\text { upgrades } \\
\text { where } \\
5>4>3>2>1\end{array}$ \\
\hline Age & Age & .622 \\
& Pearson Correlation & 1 & .074 \\
& Sig. (2-tailed) & & 9 \\
$\mathrm{~N}$ & Pearson Correlation & .622 & 1 \\
\hline upgrades where & Sig. (2-tailed) & .074 & \\
$5>4>3>2>1$ &
\end{tabular}




\begin{tabular}{|ll|r|r|}
\hline & & \multicolumn{1}{c|}{$\begin{array}{c}\text { upgrades } \\
\text { where } \\
5>4>3>2>1\end{array}$} \\
\hline Age & Age & .622 \\
& Pearson Correlation & 1 & .074 \\
& Sig. (2-tailed) & & 9 \\
N & 9 & 1 \\
\hline upgrades where & Pearson Correlation & .622 & 9 \\
\hline $5>4>3>2>1$ & Sig. (2-tailed) & .074 & \\
& $\mathrm{~N}$ & 9 & \\
\hline
\end{tabular}

There is strong correlation between Age and Upgrades where $5>4>3>2>1$.

There is no significant difference.

Correlations

\begin{tabular}{|ll|r|r|}
\hline & & Age & \multicolumn{1}{|c|}{$\begin{array}{c}\text { services likes } \\
\text { the most }\end{array}$} \\
\hline Age & Pearson Correlation & 1 & .524 \\
& Sig. (2-tailed) & & .148 \\
& $\mathrm{~N}$ & 9 & 9 \\
\hline services likes the most & Pearson Correlation & .524 & 1 \\
& Sig. (2-tailed) & .148 & 9 \\
& $\mathrm{~N}$ & 9 & 9 \\
\hline
\end{tabular}

There is strong positive correlation between Age and Services likes the most.

There is no significant difference.

\section{Correlations}

\begin{tabular}{|ll|r|r|}
\hline & & \multicolumn{1}{|c|}{$\begin{array}{c}\text { Preferred } \\
\text { decision making } \\
\text { factors }\end{array}$} \\
\hline Age & Pearson Correlation & 1 & .286 \\
& Sig. (2-tailed) & 9 & .455 \\
& $\mathrm{~N}$ & .286 & 9 \\
\hline Preferred decision making & Pearson Correlation & .455 & 1 \\
factors & Sig. (2-tailed) & 9 & 9 \\
& $\mathrm{~N}$ & & \\
& & 9 & \\
\hline
\end{tabular}

Conclusion;- There is positive correlation between Age and preferred decision making factors. There is no significant difference.

Table :- 2.4

Correlations

\begin{tabular}{|c|c|c|c|}
\hline & & Age & $\begin{array}{l}\text { high combined } \\
\text { cycle efficiency }\end{array}$ \\
\hline \multirow[t]{3}{*}{ Age } & Pearson Correlation & 1 & $.909^{* * *}$ \\
\hline & Sig. (2-tailed) & & .001 \\
\hline & $\mathrm{N}$ & 9 & 9 \\
\hline
\end{tabular}




\begin{tabular}{|ll|r|r|}
\hline high combined cycle & Pearson Correlation & $.909^{* *}$ & 1 \\
efficiency & Sig. (2-tailed) & .001 & \\
& $\mathrm{~N}$ & 9 & 9 \\
\hline
\end{tabular}

**. Correlation is significant at the 0.01 level (2-tailed).

There is strong positive correlation between Age and High combined cycle efficiency.

There is significant difference.

Table :- 2.5

ANOVA

\begin{tabular}{|l|r|r|r|r|r|}
\cline { 2 - 6 } \multicolumn{1}{l|}{ Age } & & & & & \\
\hline & Sum of Squares & df & Mean Square & F & Sig. \\
\hline Between Groups & 5.689 & 1 & 5.689 & 33.185 & .001 \\
Within Groups & 1.200 & 7 & .171 & & \\
Total & 6.889 & 8 & & & \\
\hline
\end{tabular}

Conclusion:- There is significant difference among the perception in the age group, $<30,30-45 \&>45$, respondents in their choice of "High combined cycle efficiency" as the key driver for O\&M(Operation \& maintenance) for their plant

Correlations

\begin{tabular}{|ll|r|r|}
\hline & & \multicolumn{2}{|c|}{$\begin{array}{c}\text { Low NOx } \\
\text { emission }\end{array}$} \\
\hline Age & Pearson Correlation & 1 & .180 \\
& Sig. (2-tailed) & & .644 \\
& $\mathrm{~N}$ & 9 & 9 \\
\hline Low NOx emission & Pearson Correlation & .180 & 1 \\
& Sig. (2-tailed) & .644 & 9 \\
& $\mathrm{~N}$ & 9 & \\
& & & \\
&
\end{tabular}

Conclusion:- There is positive correlation between Age and Low NOx emission.

There is no significant difference.

Table:- 2.6

Correlations

\begin{tabular}{|c|c|c|c|}
\hline & & Age & $\begin{array}{c}\text { low specific cost } \\
(\text { per KW) }\end{array}$ \\
\hline \multirow[t]{3}{*}{ Age } & Pearson Correlation & 1 & $.898^{* *}$ \\
\hline & Sig. (2-tailed) & & .001 \\
\hline & $\mathrm{N}$ & 9 & 9 \\
\hline \multirow[t]{3}{*}{ low specific cost (per KW) } & Pearson Correlation & $.898^{* * *}$ & 1 \\
\hline & Sig. (2-tailed) & .001 & \\
\hline & $\mathrm{N}$ & 9 & 9 \\
\hline
\end{tabular}

**. Correlation is significant at the 0.01 level (2-tailed).

Conclusion:- There is strong positive correlation between Age and

Low specific cost (per KW).

There is significant difference. 
Table :- 2.7

\begin{tabular}{|c|c|c|c|c|c|}
\hline \multirow[b]{2}{*}{ Age } & \multicolumn{4}{|c|}{ ANOVA } & \\
\hline & & & & & \\
\hline & Sum of Squares & $\mathrm{df}$ & Mean Square & $\mathrm{F}$ & Sig. \\
\hline Between Groups & 5.556 & 1 & 5.556 & 29.167 & .001 \\
\hline Within Groups & 1.333 & 7 & .190 & & \\
\hline Total & 6.889 & 8 & & & \\
\hline
\end{tabular}

Conclusion:- There is significant difference among the perception in the age group, <30, 30-45 \&>45, respondents in their choice of "Low specific cost(per KW)" as the key driver for O\&M(Operation \& maintenance) for their plant

Table :- 2.8

\section{Correlations}

\begin{tabular}{|ll|r|r|}
\hline & Age & High availability \\
\hline Age & Pearson Correlation & 1 & $.898^{* * *}$ \\
& Sig. (2-tailed) & & .001 \\
& $\mathrm{~N}$ & 9 & 9 \\
\hline High availability & Pearson Correlation & $.898^{* *}$ & 1 \\
& Sig. (2-tailed) & .001 & \\
& $\mathrm{~N}$ & 9 & 9 \\
\hline
\end{tabular}

**. Correlation is significant at the 0.01 level (2-tailed).

Conclusion:- There is strong positive correlation between Age and high availability.

There is significant difference.

Table 2.9

\begin{tabular}{|c|c|c|c|c|c|}
\multicolumn{1}{c|}{ ANOVA } & & & \\
\cline { 2 - 6 } \multicolumn{1}{c|}{ Age } & Sum of Squares & df & Mean Square & F & Sig. \\
\hline Between Groups & 5.556 & 1 & 5.556 & 29.167 & .001 \\
Within Groups & 1.333 & 7 & .190 & & \\
Total & 6.889 & 8 & & & \\
\hline
\end{tabular}

Conclusion:- There is significant difference among the perception in the age group, $<30,30-45 \&>45$, respondents in their choice of "High availability" as the key driver for O\&M(Operation \& maintenance) for their plant

\begin{tabular}{|ll|r|r|}
\hline \multicolumn{2}{|c|}{ Correlations } \\
\hline & Age & $\begin{array}{r}\text { operating } \\
\text { flexibility }\end{array}$ \\
\hline Age & Pearson Correlation & 1 & .653 \\
& Sig. (2-tailed) & & .056 \\
& $\mathrm{~N}$ & 9 & 9 \\
\hline operating flexibility & Pearson Correlation & .653 & 1 \\
& Sig. (2-tailed) & .056 & 9 \\
& $\mathrm{~N}$ & 9 & \\
\hline
\end{tabular}

Conclusion:- There is strong positive correlation between age and operating flexibility.

There is no significant difference. 


\begin{tabular}{|ll|r|r|}
\hline \multicolumn{2}{|c|}{ Correlations } \\
\hline Age & Age & High reliability \\
\hline & Pearson Correlation & 1 & .625 \\
& Sig. (2-tailed) & & .072 \\
& $\mathrm{~N}$ & 9 & 9 \\
\hline High reliability & Pearson Correlation & .625 & 1 \\
& Sig. (2-tailed) & .072 & 9 \\
& $\mathrm{~N}$ & 9 & 9 \\
\hline
\end{tabular}

Conclusion:- there is strong positive correlation between Age and High reliability.

There is no significant difference.

Table :- 3

\section{Correlations}

\begin{tabular}{|ll|r|r|}
\hline & Age & $\begin{array}{c}\text { High simple } \\
\text { cycle efficiency }\end{array}$ \\
\hline Age & Pearson Correlation & 1 & $.898^{* *}$ \\
& Sig. (2-tailed) & & .001 \\
$\mathrm{~N}$ & 9 & 9 \\
\hline High simple cycle efficiency & Pearson Correlation & $.898^{* *}$ & 1 \\
& Sig. (2-tailed) & .001 & 9 \\
$\mathrm{~N}$ & 9 & 9 \\
\hline
\end{tabular}

**. Correlation is significant at the 0.01 level (2-tailed).

Conclusion:- There is strong positive correlation between Age and High simple cycle efficiency. There is significant difference.

Table :- 3.1

\begin{tabular}{|c|c|c|c|c|c|}
\hline \multirow[b]{2}{*}{ Age } & \multicolumn{4}{|c|}{ ANOVA } & \\
\hline & & & & & \\
\hline & Sum of Squares & $\mathrm{df}$ & Mean Square & $\mathrm{F}$ & Sig. \\
\hline Between Groups & 5.556 & 1 & 5.556 & 29.167 & .001 \\
\hline Within Groups & 1.333 & 7 & 190 & & \\
\hline Total & 6.889 & 8 & & & \\
\hline
\end{tabular}

Conclusion:- There is significant difference among the perception in the age group, $<30,30-45 \&>45$, respondents in their choice of "High simple cycle efficiency" as the key driver for O\&M(Operation \& maintenance) for their plant

\begin{tabular}{|c|c|c|c|}
\hline \multicolumn{4}{|c|}{ Correlations } \\
\hline & & Age & $\begin{array}{c}\text { Cooling } \\
\text { Technology }\end{array}$ \\
\hline \multirow[t]{3}{*}{ Age } & Pearson Correlation & 1 & .653 \\
\hline & Sig. (2-tailed) & & .056 \\
\hline & $\mathrm{N}$ & 9 & 9 \\
\hline \multirow[t]{3}{*}{ Cooling Technology } & Pearson Correlation & .653 & 1 \\
\hline & Sig. (2-tailed) & .056 & \\
\hline & $\mathrm{N}$ & 9 & 9 \\
\hline
\end{tabular}

Conclusion:- There is strong positive correlation between Age and cooling technology.

There is no significant difference. 


\begin{tabular}{|ll|r|r|}
\hline \multicolumn{1}{|c|}{ Correlations } \\
\hline Age & Age & \multicolumn{1}{c|}{$\begin{array}{c}\text { Firing } \\
\text { temperature }\end{array}$} \\
\hline & Pearson Correlation & 1 & .653 \\
& Sig. (2-tailed) & & .056 \\
& $\mathrm{~N}$ & 9 & 9 \\
\hline Firing temperature & Pearson Correlation & .653 & 1 \\
& Sig. (2-tailed) & .056 & 9 \\
& $\mathrm{~N}$ & 9 & \\
\hline
\end{tabular}

Conclusion:- There is strong correlation between Age and Firing temperature.

There is no significant difference.

Table:- 3.2

\section{Correlations}

\begin{tabular}{|ll|r|r|}
\hline & & Age & Pressure ratio \\
\hline Age & Pearson Correlation & 1 & $.898^{* * *}$ \\
& Sig. (2-tailed) & & .001 \\
& $\mathrm{~N}$ & 9 & 9 \\
\hline Pressure ratio & Pearson Correlation & $.898^{* *}$ & 1 \\
& Sig. (2-tailed) & .001 & 9 \\
& $\mathrm{~N}$ & 9 & 9 \\
\hline
\end{tabular}

**. Correlation is significant at the 0.01 level (2-tailed).

There is strong positive correlation between Age and pressure ratio.

There is significant difference.

Table :- 3.3

\begin{tabular}{|l|r|r|r|r|r|}
\multicolumn{1}{c|}{ ANO } & & & & & \\
\cline { 2 - 6 } & Sum of Squares & df & Mean Square & F & Sig. \\
\hline Between Groups & 5.556 & 1 & 5.556 & 29.167 & .001 \\
Within Groups & 1.333 & 7 & .190 & & \\
Total & 6.889 & 8 & & & \\
\hline
\end{tabular}

Conclusion:- There is significant difference among the perception in the age group, <30, 30-45 \&>45, respondents in their choice of "Pressure ratio" as the key driver for O\&M(Operation \& maintenance) for their plant

\section{Correlations}

\begin{tabular}{|ll|r|r|}
\hline & & \multicolumn{1}{|c|}{ Age } & Mass flow \\
\hline Age & Pearson Correlation & 1 & .561 \\
& Sig. (2-tailed) & & .116 \\
& $\mathrm{~N}$ & 9 & 9 \\
\hline Mass flow & Pearson Correlation & .561 & 1 \\
& Sig. (2-tailed) & .116 & \\
$\mathrm{~N}$ & 9 & 9 \\
\hline
\end{tabular}

Conclusion:- There is strong positive correlation between Age and Mass flow.

There is no significant difference. 


\begin{tabular}{|ll|r|r|}
\hline & & \\
\hline & Correlations & $\begin{array}{c}\text { Planned outage } \\
\text { frequency }\end{array}$ \\
\hline Age & Pearson Correlation & 1 & .311 \\
& Sig. (2-tailed) & & .415 \\
& $\mathrm{~N}$ & 9 & 9 \\
\hline Planned outage frequency & Pearson Correlation & .311 & 1 \\
& Sig. (2-tailed) & .415 & 9 \\
& $\mathrm{~N}$ & 9 & 9 \\
\hline
\end{tabular}

Conclusion:- there is positive correlation between Age and planned outage frequency. There is no significant difference.

\section{Correlations}

\begin{tabular}{|ll|r|r|}
\hline & & Age & Outage duration \\
\hline Age & Pearson Correlation & 1 & .180 \\
& Sig. (2-tailed) & & .644 \\
& $\mathrm{~N}$ & 9 & 9 \\
\hline Outage duration & Pearson Correlation & .180 & 1 \\
& Sig. (2-tailed) & .644 & \\
& $\mathrm{~N}$ & 9 & 9 \\
\hline
\end{tabular}

There is positive correlation between Age and outage duration. There is no significant difference.

\begin{tabular}{|ll|r|r|}
\hline & Correlations & \multicolumn{1}{c|}{$\begin{array}{c}\text { Parts } \\
\text { replacement } \\
\text { frequency }\end{array}$} \\
\hline Age & Age & 1 & -.498 \\
& Pearson Correlation & & .173 \\
& Sig. (2-tailed) & 9 & 9 \\
\hline Parts replacement & $\mathrm{N}$ & -.498 & 1 \\
frequency & Pearson Correlation & .173 & 9 \\
& Sig. (2-tailed) & 9 & \\
& $\mathrm{~N}$ & \multicolumn{2}{|c}{} \\
\end{tabular}

Conclusion:- There is negative correlation between age and Parts replacement frequency. There is no significant difference.

\begin{tabular}{|ll|r|r|}
\hline \multicolumn{1}{|c|}{ Correlations } & \multicolumn{2}{c|}{$\begin{array}{c}\text { Parts } \\
\text { replacement } \\
\text { costs }\end{array}$} \\
\hline Age & Age & 1 & .449 \\
& Pearson Correlation & & .225 \\
& Sig. (2-tailed) & 9 & 9 \\
& $\mathrm{~N}$ & .449 & 1 \\
\hline Parts replacement costs & Pearson Correlation & .225 & 9 \\
& Sig. (2-tailed) & 9 & \\
& $\mathrm{~N}$ & & 9
\end{tabular}


Conclusion:- There is positive correlation between age and parts replacement costs.

There is no significant difference.

\begin{tabular}{|c|c|c|c|}
\hline \multicolumn{4}{|c|}{ Correlations } \\
\hline & & Age & $\begin{array}{c}\text { Uncertainty } \\
\text { repair/maintenan } \\
\text { ce }\end{array}$ \\
\hline \multirow[t]{3}{*}{ Age } & Pearson Correlation & 1 & .238 \\
\hline & Sig. (2-tailed) & & .538 \\
\hline & $\mathrm{N}$ & 9 & 9 \\
\hline \multirow{3}{*}{$\begin{array}{l}\text { Uncertainty } \\
\text { repair/maintenance }\end{array}$} & Pearson Correlation & .238 & 1 \\
\hline & Sig. (2-tailed) & .538 & \\
\hline & $\mathrm{N}$ & 9 & 9 \\
\hline
\end{tabular}

Conclusion:- There is positive correlation between age and uncertainty repair/maintenance. There is no significant difference.

\begin{tabular}{|ll|r|r|}
\hline \multicolumn{1}{|c|}{ Correlations } & & \multicolumn{2}{c|}{$\begin{array}{c}\text { daily operation } \\
\text { costs }\end{array}$} \\
\hline Age & Age & 1 & .373 \\
& Searson Correlation & & .322 \\
& Sig. (2-tailed) & 9 & 9 \\
& $\mathrm{~N}$ & .373 & 1 \\
\hline daily operation costs & Pearson Correlation & .322 & 9 \\
& Sig. (2-tailed) & 9 & \\
& $\mathrm{~N}$ & & \\
\end{tabular}

Conclusion:- there is positive correlation between age and daily operation cost.

There is no significant difference.

\begin{tabular}{|ll|r|r|}
\hline \multicolumn{1}{|c|}{ Correlations } \\
\hline Age & Age & NOx abatement \\
& Pig. (2-tailed) & 1 & .445 \\
& $\mathrm{~N}$ & 9 & .231 \\
& NOx abatement & 9 \\
& Pearson Correlation & .445 & 1 \\
& Sig. (2-tailed) & .231 & \\
& $\mathrm{~N}$ & 9 & 9 \\
\hline
\end{tabular}

Conclusion:- There is positive correlation between Age and NOx abatement.

There is no significant difference.

Correlations

\begin{tabular}{|ll|r|r|}
\hline & & Age & $\begin{array}{c}\text { Long time surety } \\
\text { of price }\end{array}$ \\
\hline Age & Pearson Correlation & 1 & -.508 \\
& Sig. (2-tailed) & & .163 \\
& $\mathrm{~N}$ & 9 & 9 \\
\hline Long time surety of price & Pearson Correlation & -.508 & 1 \\
& Sig. (2-tailed) & .163 & \\
\hline
\end{tabular}




\begin{tabular}{|ll|r|r|}
\hline \multicolumn{1}{|c|}{ Correlations } & \\
\hline Age & Age & $\begin{array}{c}\text { Long time surety } \\
\text { of price }\end{array}$ \\
& Sig. (2-tailed) & 1 & -.508 \\
& $\mathrm{~N}$ & 9 & .163 \\
\hline Long time surety of price & Pearson Correlation & -.508 & 9 \\
& Sig. (2-tailed) & .163 & 1 \\
& $\mathrm{~N}$ & 9 & 9 \\
\hline
\end{tabular}

Conclusion:- There is negative correlation between age and long time surety of price.

There is no significant difference.

\section{Correlations}

\begin{tabular}{|ll|r|r|}
\hline & Age & $\begin{array}{c}\text { Provision of high } \\
\text { quality outage } \\
\text { services }\end{array}$ \\
\hline Age & Pearson Correlation & 1 & -.318 \\
& Sig. (2-tailed) & 9 & .405 \\
& $\mathrm{~N}$ & -.318 & 9 \\
\hline Provision of high quality & Pearson Correlation & .405 & 1 \\
outage services & Sig. (2-tailed) & 9 & 9 \\
& $\mathrm{~N}$ & & \\
\hline
\end{tabular}

Conclusion :- There is negative correlation between age and provision of high quality outage services. There is no significant difference.

\section{Correlations}

\begin{tabular}{|c|c|c|c|}
\hline & & Age & $\begin{array}{c}\text { Local project } \\
\text { management \& } \\
\text { execution }\end{array}$ \\
\hline \multirow[t]{3}{*}{ Age } & Pearson Correlation & 1 & -.653 \\
\hline & Sig. (2-tailed) & & .056 \\
\hline & $\mathrm{N}$ & 9 & 9 \\
\hline \multirow{3}{*}{$\begin{array}{l}\text { Local project management } \\
\& \text { execution }\end{array}$} & Pearson Correlation & -.653 & 1 \\
\hline & Sig. (2-tailed) & .056 & \\
\hline & $\mathrm{N}$ & 9 & 9 \\
\hline
\end{tabular}

Conclusion:- There is negative correlation between age and local project management $\&$ execution. There is no significant difference.

\begin{tabular}{|c|c|c|c|}
\hline \multicolumn{4}{|c|}{ Correlations } \\
\hline & & Age & $\begin{array}{c}\text { Rapid supply of } \\
\text { key parts in case } \\
\text { of emergency } \\
\text { failures }\end{array}$ \\
\hline Age & $\begin{array}{l}\text { Pearson Correlation } \\
\text { Sig. (2-tailed) } \\
\mathrm{N}\end{array}$ & $\begin{array}{l}1 \\
9\end{array}$ & 9 \\
\hline
\end{tabular}




\begin{tabular}{|l|r|r|}
\hline $\begin{array}{l}\text { Rapid supply of key parts in Pearson Correlation } \\
\text { case of emergency failures } \\
\text { Sig. (2-tailed) }\end{array}$ & $\cdot$ &. \\
$\mathrm{N}$ & 9 & 9 \\
\hline
\end{tabular}

a. Cannot be computed because at least one of the variables is constant.

\section{Correlations}

\begin{tabular}{|c|c|c|c|}
\hline & & Age & $\begin{array}{c}\text { Remote expert } \\
\text { monitoring of } \\
\text { plant }\end{array}$ \\
\hline \multirow[t]{3}{*}{ Age } & Pearson Correlation & 1 & -.127 \\
\hline & Sig. (2-tailed) & & .745 \\
\hline & $\mathrm{N}$ & 9 & 9 \\
\hline \multirow{3}{*}{$\begin{array}{l}\text { Remote expert monitoring of } \\
\text { plant }\end{array}$} & Pearson Correlation & -.127 & 1 \\
\hline & Sig. (2-tailed) & .745 & \\
\hline & $\mathrm{N}$ & 9 & 9 \\
\hline
\end{tabular}

Conclusion:- there is negative correlation between age and remote expert monitoring of plant. There is no significant difference.

\section{Correlations}

\begin{tabular}{|ll|r|r|}
\hline & & & $\begin{array}{c}\text { An on site expert } \\
\text { to provide } \\
\text { immediate } \\
\text { advice } \\
\text { \&coordinate } \\
\text { activities }\end{array}$ \\
\hline Age & Pearson Correlation & 1 & -.045 \\
& Sig. (2-tailed) & Age & .909 \\
An on site expert to provide & Pearson Correlation & -.045 & 9 \\
immediate advice & Sig. (2-tailed) & .909 & 1 \\
\&coordinate activities & $\mathrm{N}$ & 9 & 9 \\
\hline
\end{tabular}

Conclusion:- There is negative correlation between age and An on site expert to provide immediate advice and coordinate activities.

There is no significant difference.

\section{Correlations}

\begin{tabular}{|ll|r|r|}
\hline & & Age & $\begin{array}{r}\text { Fuel system } \\
\text { conversions }\end{array}$ \\
\hline Age & Pearson Correlation & 1 & -.509 \\
& Sig. (2-tailed) & .162 \\
& $\mathrm{~N}$ & 9 & 9 \\
\hline Fuel system conversions & Pearson Correlation & -.509 & 1 \\
& Sig. (2-tailed) & .162 & 9 \\
& $\mathrm{~N}$ & 9 & \\
\hline
\end{tabular}

Conclusion:-There is negative correlation between age and Fuel system conversions.

There is no significant difference. 


\begin{tabular}{|ll|r|r|}
\hline \multicolumn{1}{|c|}{ Correlations } & \multicolumn{1}{c|}{$\begin{array}{c}\text { Emission } \\
\text { abatement } \\
\text { systems }\end{array}$} \\
\hline Age & Age & 1 & -.602 \\
& Pearson Correlation & & .086 \\
& Sig. (2-tailed) & 9 & 9 \\
$\mathrm{~N}$ & -.602 & 1 \\
\hline Emission abatement systems & Pearson Correlation & .086 & 9 \\
& Sig. (2-tailed) & 9 & 9 \\
& $\mathrm{~N}$ &
\end{tabular}

Conclusion :- There is negative correlation between age and emission abatement systems. There is no significant difference

\section{Correlations}

\begin{tabular}{|ll|r|r|}
\hline & Age & $\begin{array}{c}\text { Auxiliary system } \\
\text { modification }\end{array}$ \\
\hline Age & Pearson Correlation & 1 & -.521 \\
& Sig. (2-tailed) & & .150 \\
& $\mathrm{~N}$ & 9 & 9 \\
\hline Auxiliary system & Pearson Correlation & -.521 & 1 \\
modification & Sig. (2-tailed) & .150 & 9 \\
& $\mathrm{~N}$ & 9 & 9 \\
\hline
\end{tabular}

Conclusion :- There is negative correlation between age and Auxiliary system modification. There is no significant difference.

\begin{tabular}{|ll|r|r|}
\hline & Correlations & & $\begin{array}{c}\text { Instrumentation } \\
\& \text { control } \\
\text { system } \\
\text { modification }\end{array}$ \\
\hline Age & Pearson Correlation & 1 & -.518 \\
& Sig. (2-tailed) & & .153 \\
& $\mathrm{~N}$ & 9 & 9 \\
\hline Instrumentation \& control & Pearson Correlation & -.518 & 1 \\
system modification & Sig. (2-tailed) & .153 & 9 \\
\hline
\end{tabular}

Conclusion:- There is negative correlation between age and Instrumentation and control system modification. There is no significant difference.

Correlations

\begin{tabular}{|ll|r|r|}
\hline & Age & $\begin{array}{c}\text { Corrosion } \\
\text { monitoring }\end{array}$ \\
\hline Age & Pearson Correlation & 1 & .340 \\
& Sig. (2-tailed) & & .371 \\
& $\mathrm{~N}$ & 9 & 9 \\
\hline Corrosion monitoring & Pearson Correlation & .340 & 1 \\
& Sig. (2-tailed) & .371 &
\end{tabular}




\begin{tabular}{|ll|r|r|}
\hline \multicolumn{2}{|c|}{ Correlations } & \multicolumn{1}{c|}{$\begin{array}{c}\text { Corrosion } \\
\text { monitoring }\end{array}$} \\
\hline Age & Age & 1 & .340 \\
& Pearson Correlation & & .371 \\
& Sig. (2-tailed) & 9 & 9 \\
& $\mathrm{~N}$ & .340 & 1 \\
\hline Corrosion monitoring & Pearson Correlation & .371 & 9 \\
& Sig. (2-tailed) & 9 & \\
& $\mathrm{~N}$ & & \\
&
\end{tabular}

Conclusion:- There is positive correlation between age and corrosion monitoring. There is no significant difference.

\section{Correlations}

\begin{tabular}{|ll|r|r|}
\hline & & Age & Air sampling \\
\hline Age & Pearson Correlation & 1 & -.014 \\
& Sig. (2-tailed) & & .971 \\
& $\mathrm{~N}$ & 9 & 9 \\
\hline Air sampling & Pearson Correlation & -.014 & 1 \\
& Sig. (2-tailed) & .971 & \\
& $\mathrm{~N}$ & 9 & 9 \\
\hline
\end{tabular}

Conclusion:- There is negative correlation between age and Air sampling. There is no significant difference.

\section{Correlations}

\begin{tabular}{|ll|r|r|}
\hline & Age & Material testing \\
\hline Age & Pearson Correlation & 1 & .096 \\
& Sig. (2-tailed) & & .806 \\
& $\mathrm{~N}$ & 9 & 9 \\
\hline Material testing & Pearson Correlation & .096 & 1 \\
& Sig. (2-tailed) & .806 & \\
& $\mathrm{~N}$ & 9 & 9 \\
\hline
\end{tabular}

Conclusion:- There is positive correlation between age and material testing.

There is no significant difference.

\section{Correlations}

\begin{tabular}{|ll|r|r|}
\hline & & \multicolumn{1}{|c|}{ Age } & Site surveys \\
\hline Age & Pearson Correlation & 1 & -.129 \\
& Sig. (2-tailed) & & .741 \\
& $\mathrm{~N}$ & 9 & 9 \\
\hline Site surveys & Pearson Correlation & -.129 & 1 \\
& Sig. (2-tailed) & .741 & \\
& $\mathrm{~N}$ & 9 & 9 \\
\hline
\end{tabular}

Conclusion:- There is negative correlation between age and site surveys. There is significant difference.

\section{Correlations}




\begin{tabular}{|ll|r|r|}
\hline & Age & \multicolumn{2}{|c|}{$\begin{array}{c}\text { Technical } \\
\text { advisory } \\
\text { services }\end{array}$} \\
\hline Age & Pearson Correlation & 1 & .287 \\
& Sig. (2-tailed) & 9 & .453 \\
& $\mathrm{~N}$ & .287 & 9 \\
\hline Technical advisory services & Pearson Correlation & .453 & 9 \\
& Sig. (2-tailed) & 9 & 9 \\
& $\mathrm{~N}$ & & 9 \\
\hline
\end{tabular}

Conclusion:-There is positive correlation between age and technical advisory services.

There is no significant difference.

\begin{tabular}{|ll|r|r|}
\hline \multicolumn{1}{|c|}{ Correlations } & & $\begin{array}{c}\text { Your next order } \\
\text { will be to } \\
\text { BGGTS }\end{array}$ \\
\hline Age & Age & 1 & .467 \\
& Pearson Correlation & & .205 \\
& Sig. (2-tailed) & 9 & 9 \\
\hline N & Pearson Correlation & .467 & 1 \\
BGGTS & Sig. (2-tailed) & .205 & 9 \\
& N & 9 & \\
\hline
\end{tabular}

Conclusion:- There is positive correlation between age and Your next order will be to BGGTS. There is no significant difference.

\section{Correlations}

\begin{tabular}{|ll|r|r|}
\hline & Age & \multicolumn{2}{|c|}{$\begin{array}{c}\text { Stisfied by } \\
\text { services } \\
\text { delivered }\end{array}$} \\
\hline Age & Pearson Correlation & 1 & .572 \\
& Sig. (2-tailed) & 9 & .108 \\
& $\mathrm{~N}$ & .572 & 9 \\
\hline Stisfied by services & Pearson Correlation & .108 & 1 \\
delivered & Sig. (2-tailed) & 9 & 9 \\
& $\mathrm{~N}$ & 9 \\
\hline
\end{tabular}

Conclusion:- There is strong positive correlation between age and satisfied by services delivered. There is no significant difference.

\section{Correlations}

\begin{tabular}{|ll|r|r|}
\hline & Age & $\begin{array}{c}\text { Services worth } \\
\text { money paid }\end{array}$ \\
\hline Age & Pearson Correlation & 1 & .453 \\
& Sig. (2-tailed) & & .220 \\
& $\mathrm{~N}$ & 9 & 9 \\
\hline Services worth money paid & Pearson Correlation & .453 & 1 \\
& Sig. (2-tailed) & .220 & 9 \\
& $\mathrm{~N}$ & 9 & 9 \\
\hline
\end{tabular}


Conclusion:- There is positive correlation between age and services worth money paid.

There is no significant difference

\section{Correlations}

\begin{tabular}{|ll|r|r|}
\hline & & $\begin{array}{c}\text { BGGTS } \\
\text { response time is } \\
\text { satisfactory }\end{array}$ \\
\hline Age & Pearson Correlation & 1 & .340 \\
& Sig. (2-tailed) & 9 & .371 \\
& $\mathrm{~N}$ & .340 & 9 \\
\hline BGGTS response time is & Pearson Correlation & .371 & 1 \\
satisfactory & Sig. (2-tailed) & 9 & 9 \\
& $\mathrm{~N}$ & 9 & 9 \\
\hline
\end{tabular}

Conclusion:- There is strong correlation between age and BGGTS response time is satisfactory. There is no significant difference.

\section{Correlations}

\begin{tabular}{|ll|r|r|}
\hline & Age & $\begin{array}{c}\text { Technical } \\
\text { support is } \\
\text { satisfactory }\end{array}$ \\
\hline Age & Pearson Correlation & 1 & .539 \\
& Sig. (2-tailed) & 9 & .134 \\
& $\mathrm{~N}$ & .539 & 9 \\
\hline Technical support is & Pearson Correlation & .134 & 1 \\
satisfactory & Sig. (2-tailed) & 9 & 9 \\
& $\mathrm{~N}$ & & 9 \\
\hline
\end{tabular}

Conclusion:- There is Strong positive correlation between age and technical support is satisfactory.

There is no significance difference.

\section{Findings:-}

\begin{tabular}{|c|c|}
\hline H1 & ir choice of "Service" as the critical parameter for after sales service for their plant \\
\hline $\mathrm{H} 2$ & $\begin{array}{l}<30,30-45 \&>45, \text { re } \\
\text { vice for their plant }\end{array}$ \\
\hline H3 & $\begin{array}{l}\text { ip, }<30,30-45 \&>45 \text {, respondents in } \\
\text { es service for their plant }\end{array}$ \\
\hline $\mathrm{H} 4$ & $\begin{array}{l}\mathrm{p},<30,30-45 \&>45,1 \\
\text { or O\&M(Operation \& }\end{array}$ \\
\hline H5 & $\begin{array}{l}\mathrm{p},<30,30-45 \&>45, \mathrm{r} \\
\& \mathrm{M}(\text { Operation \& mai }\end{array}$ \\
\hline H6 & $<30,30-45 \&>45$ \\
\hline H7 & $\begin{array}{l}\text { There is significant difference among the association in the age group, }<30,30-45 \&>45 \text {, respon } \\
\text { their choice of "High simple cycle efficiency" as the key driver for O\&M(Operation \& maintena } \\
\text { their plant }\end{array}$ \\
\hline & \\
\hline
\end{tabular}


Suggestion:-

\begin{tabular}{|l|l|}
\hline Parameters & Facts \\
\hline Service & the critical parameter for after sales service for their plant \\
\hline Maintenance & the critical parameter for after sales service for their plant \\
\hline Online support & the critical parameter for after sales service for their plant \\
\hline High combined cycle efficiency & the key driver for O\&M(Operation \& maintenance) for their plant \\
\hline Low specific cost(per KW) & the key driver for O\&M(Operation \& maintenance) for their plant \\
\hline High availability & the key driver for O\&M(Operation \& maintenance) for their plant \\
\hline High simple cycle efficiency & the key driver for O\&M(Operation \& maintenance) for their plant \\
\hline Pressure ratio & the key driver for O\&M(Operation \& maintenance) for their plant \\
\hline
\end{tabular}

\section{Conclusion: -}

BGGTS customers are satisfied in most of the aspects being tested. There is difference in the perception of customers on the basis of age group, Customers greater than 45 years age group seems to be more satisfied then customers in the age group 30-45, where as customers smaller than 30 years of age groups are sometime neutral in their response but in most of the case their perceptions are matching with customers in the age group greater than 45 .

Weighted Average Method

\begin{tabular}{|c|c|c|c|c|c|c|c|c|}
\hline & $\begin{array}{l}\text { Strongly } \\
\text { agree }\end{array}$ & Agree & $\begin{array}{l}\text { Neutr } \\
\text { al }\end{array}$ & Disagree & $\begin{array}{l}\text { Strongl } \\
\text { y } \\
\text { disagre } \\
\text { e }\end{array}$ & Total & $\begin{array}{l}\text { Weighted } \\
\text { average }\end{array}$ & Rank \\
\hline & 5 & 4 & 3 & 2 & 1 & & & \\
\hline $\begin{array}{l}\text { High } \\
\text { combined } \\
\text { cycle } \\
\text { efficiency }\end{array}$ & 4 & 5 & 0 & 0 & 0 & 40 & 2.666 & \\
\hline $\begin{array}{l}\text { Low NOx } \\
\text { emission }\end{array}$ & 3 & 6 & 0 & 0 & 0 & 39 & 2.6 & \\
\hline $\begin{array}{l}\text { Low specific } \\
\text { cost (Per KW) }\end{array}$ & 6 & 3 & 0 & 0 & 0 & 42 & 2.8 & 1 \\
\hline $\begin{array}{l}\text { High } \\
\text { availability }\end{array}$ & 6 & 3 & 0 & 0 & 0 & 42 & 2.8 & 1 \\
\hline $\begin{array}{l}\text { Operating } \\
\text { flexibility }\end{array}$ & 4 & 5 & 0 & 0 & 0 & 40 & 2.666 & \\
\hline $\begin{array}{l}\text { High } \\
\text { reliability }\end{array}$ & 5 & 4 & 0 & 0 & 0 & 41 & 2.733 & \\
\hline $\begin{array}{l}\text { High simple } \\
\text { cycle } \\
\text { efficiency }\end{array}$ & 6 & 3 & 0 & 0 & 0 & 42 & 2.8 & 1 \\
\hline $\begin{array}{l}\text { Cooling } \\
\text { technology }\end{array}$ & 4 & 5 & 0 & 0 & 0 & 40 & 2.666 & \\
\hline $\begin{array}{l}\text { Firing } \\
\text { temperature }\end{array}$ & 4 & 5 & 0 & 0 & 0 & 40 & 2.666 & \\
\hline Pressure ratio & 6 & 3 & 0 & 0 & 0 & 42 & 2.8 & 1 \\
\hline Mass flow & 3 & 5 & 1 & 0 & 0 & 38 & 2.533 & \\
\hline
\end{tabular}


Conclusion:-1

[Low specific cost (Per KW), High availability, High simple cycle efficiency, Pressure ratio] are the first ranked, and hence the key drivers for the O\&M of their plant.

\begin{tabular}{|c|c|c|c|c|c|c|c|c|}
\hline & $\begin{array}{l}\text { Least } \\
\text { important }\end{array}$ & $\begin{array}{l}\text { Slightly } \\
\text { important }\end{array}$ & Important & $\begin{array}{l}\text { Quite } \\
\text { important }\end{array}$ & $\begin{array}{l}\text { Very } \\
\text { important }\end{array}$ & Total & $\begin{array}{l}\text { Wattage } \\
\text { average } \\
\text { mean }\end{array}$ & Rank \\
\hline & 1 & 2 & 3 & 4 & 5 & & & \\
\hline $\begin{array}{l}\text { Planned outage } \\
\text { frequency }\end{array}$ & 0 & 0 & 3 & 3 & 3 & 36 & 2.4 & \\
\hline $\begin{array}{l}\text { Outage } \\
\text { duration }\end{array}$ & 0 & 0 & 0 & 3 & 6 & 42 & 2.8 & 2 \\
\hline $\begin{array}{l}\text { Parts } \\
\text { replacement } \\
\text { frequency }\end{array}$ & 0 & 0 & 2 & 4 & 3 & 37 & 2.466 & \\
\hline $\begin{array}{l}\text { Parts } \\
\text { replacement } \\
\text { cost }\end{array}$ & 0 & 0 & 0 & 3 & 6 & 42 & 2.8 & 2 \\
\hline $\begin{array}{l}\text { Uncertainty } \\
\text { repair / } \\
\text { maintenance }\end{array}$ & 0 & 0 & 0 & 2 & 7 & 43 & 2.866 & 1 \\
\hline $\begin{array}{l}\text { Daily operation } \\
\text { costs }\end{array}$ & 0 & 0 & 2 & 1 & 6 & 40 & 2.666 & \\
\hline NOx abatement & 0 & 0 & 1 & 4 & 4 & 39 & 2.6 & \\
\hline
\end{tabular}

Conclusion:-2

Uncertainty repair / maintenance is the top O\&M cost factors, And followed by Outage duration as the next $2^{\text {nd }}$ ranked $O \& M$ cost factors.

\begin{tabular}{|l|l|l|l|l|l|l|l|l|}
\hline & $\begin{array}{l}\text { Strongly } \\
\text { agree }\end{array}$ & Agree & Neutral & Disagree & $\begin{array}{l}\text { Strongly } \\
\text { disagree }\end{array}$ & Total & $\begin{array}{l}\text { Wattage } \\
\text { average } \\
\text { mean }\end{array}$ & Rank \\
\hline & 5 & 4 & 3 & 2 & 1 & & & \\
\hline $\begin{array}{l}\text { Long time } \\
\text { surety of } \\
\text { price }\end{array}$ & 7 & 1 & 1 & 0 & 0 & 42 & 2.8 & 2 \\
\hline $\begin{array}{l}\text { Provision of } \\
\text { high quality } \\
\text { outage } \\
\text { services }\end{array}$ & 7 & 1 & 1 & 0 & 0 & 42 & 2.8 & 2 \\
\hline $\begin{array}{l}\text { Local } \\
\text { project } \\
\text { management } \\
\text { \& execution }\end{array}$ & 5 & 4 & 0 & 0 & 0 & 41 & 2.733 & \\
\hline $\begin{array}{l}\text { Rapid } \\
\text { supply of } \\
\text { key parts in } \\
\text { case of } \\
\text { emergency } \\
\text { failures }\end{array}$ & 9 & 0 & 0 & 0 & 0 & 45 & 3 & 1 \\
\hline $\begin{array}{l}\text { Remote } \\
\text { expert } \\
\text { monitoring } \\
\text { of plant }\end{array}$ & 1 & 4 & 4 & 0 & 0 & 33 & 2.2 & \\
\hline $\begin{array}{l}\text { An onsite } \\
\text { expert to } \\
\text { provide } \\
\text { immediate } \\
\text { advice \& } \\
\text { coordinate } \\
\text { activities }\end{array}$ & 3 & 5 & 1 & 0 & 0 & 38 & 2.533 & \\
\hline
\end{tabular}




\section{Conclusion:-3}

Rapid supply of key parts in case of emergency failures is the first services BGGTS needs to improve in future followed by Long time surety of price, and Provision of high quality outage services

\begin{tabular}{|c|c|c|c|c|c|c|c|c|}
\hline & $\begin{array}{l}\text { Strongly } \\
\text { agree }\end{array}$ & Agree & Neutral & Disagree & $\begin{array}{l}\text { Strongly } \\
\text { disagree }\end{array}$ & Total & $\begin{array}{l}\text { Wattage } \\
\text { average } \\
\text { mean }\end{array}$ & Rank \\
\hline & 5 & 4 & 3 & 2 & 1 & & & \\
\hline $\begin{array}{l}\text { Fuel system } \\
\text { conversions }\end{array}$ & 5 & 1 & 1 & 2 & 0 & 36 & 2.4 & 3 \\
\hline $\begin{array}{l}\text { Emission } \\
\text { abatement } \\
\text { systems }\end{array}$ & 4 & 2 & 2 & 1 & 0 & 36 & 2.4 & 3 \\
\hline $\begin{array}{l}\text { Auxiliary } \\
\text { system } \\
\text { modification }\end{array}$ & 4 & 3 & 2 & o & 0 & 38 & 2.533 & 2 \\
\hline $\begin{array}{l}\text { Fuel system } \\
\text { conversions }\end{array}$ & 5 & 2 & 2 & 0 & 0 & 39 & 2.6 & 1 \\
\hline
\end{tabular}

\section{Conclusion:-4}

[Fuel system conversion is the most important services for their plant followed by Auxiliary system modification.]

\begin{tabular}{|l|l|l|l|l|l|l|l|l|}
\hline & $\begin{array}{l}\text { Strongly } \\
\text { agree }\end{array}$ & Agree & Neutral & Disagree & $\begin{array}{l}\text { Strongly } \\
\text { disagree }\end{array}$ & Total & $\begin{array}{l}\text { Wattage } \\
\text { average } \\
\text { mean }\end{array}$ & Rank \\
\hline & 5 & 4 & 3 & 2 & 1 & & & \\
\hline $\begin{array}{l}\text { Corrosion } \\
\text { monitoring }\end{array}$ & 6 & 2 & 0 & 1 & 0 & 40 & 2.666 & 1 \\
\hline $\begin{array}{l}\text { Air } \\
\text { sampling }\end{array}$ & 4 & 3 & 1 & 1 & 0 & 37 & 2.466 & \\
\hline $\begin{array}{l}\text { Material } \\
\text { testing }\end{array}$ & 5 & 2 & 1 & 1 & 0 & 38 & 2.533 & 2 \\
\hline $\begin{array}{l}\text { Site } \\
\text { surveys }\end{array}$ & 2 & 5 & 1 & 1 & 0 & 35 & 2.333 & \\
\hline $\begin{array}{l}\text { Technical } \\
\text { advisory } \\
\text { services }\end{array}$ & 5 & 3 & 0 & 0 & 1 & 38 & 2.533 & 2 \\
\hline
\end{tabular}

Conclusion:-5

[Corrosion monitoring is the most priorities service needs to be improved followed by Material testing and Technical advisory services.]

\section{Limitation of research:-}

Research result is the outcome of data being provided by three companies "BGGTS" for which the research is being done, "IPGCL" \& "PPCL" which participated in answering the questionnaire and also providing help in validation test for the questionnaire, but outcome is limited as the number of companies participated is only two \& due to the complexity of questionnaire only mechanical back ground employee were able to fill the questionnaire, and hence number of people provided feedback is nine including three from "IPGCL" \& six from "PPCL". Had the questionnaire being circulated to more companies result would have been more perfect. Also time allocated to this project is limited to 2 months only \& most of the time is devoted to understanding the product and framing the questionnaires.

\section{Future research suggestions:-}

Those who ever are interested to follow this research, as their future reference for research project should take care of following points:-

i. Involve as many companies as you can for data collection, \& provide maximum time for data feedback

ii. Try to focus on more validated and customer concerned areas.

iii. Analyze the problem of customers first, and then only try to frame the questionnaire.

iv. Strict to the accuracy of result \& to insure that, distribute and collect the questionnaire in personal

v. Keep the details given by customers as confidential because it may affect their interrelationships. 


\section{Reference:-}

[1] Cohen, M.A.,Agrawal ,N.,\&Agrawal,V.(2006).winning in the aftermarket.Harvard business review 84(5) ,129-138.

[2] Cohen,M.A.,cull,C.,Lee,H.L.,\& Willen,D.(2000).saturns supply-chain innovation : High value in after sales service.sloan management Review(snmser).,93-101

[3] Fisher,c.\&schutta,J.T.(2003).Developing New service incorrating the voice of the customer into strategic service development .milwallkee,WI:ASQ quality Press.

[4] Govers,C.(1996).what and how about quality function deployment (QFD).International journal of production economics 46-47,575585 .

[5] Gupta,s.,hanssens,D.,Hardie,B.,kahn,w.,kumar,V.Lin,N.andsriram,N.R.S.,2006."modeling customer lifetime value ,"journal of service research ,volume 9,no.2,November.2006,pp.139-155,sage publications

[6] Akao,Y.(1990).Quality Function Deployment : Integrating customer Requirements into product design cambridge .,MA:Productivity press .

[7] Agnihothri,S.,Sivasubramaniam,N.,\&Simmons,D.(2002).Leveraging to improve field

[8] service.International Journal of service industry Management ,13(1),47-68.

\section{Questionnaire:-}

Value addition to customers, through repair of Heavy gas turbine parts, increased partnership with BGGTS (BHEL GE Gas Turbine Services)

Appendices: - Dear sir/madam a survey is conducted by "Ravi Raj", the student of V.I.T University. Pursuing my 4th semester M.B.A, please support me by filling the questionnaire for "Study on the customer satisfaction on after sales services by BGGTS ". All the information's provided by you will be kept as a confidential by me.

* Required

1 Name *

2 Company name *

$3 \mathrm{Sex} *$ Male $\quad$ Female

4. Age $* C<0 \quad \bigcirc 30-45 \quad \bigcirc>45$

5 Which of the following after sales services parameters is critical for your plant? (Rate on 5 point scale)*

\begin{tabular}{|c|c|c|c|c|c|}
\hline & 1 & 2 & 3 & 4 & 5 \\
\hline Service & $r$ & $r$ & $\mathrm{C}$ & $c$ & $c$ \\
\hline Documentation & $r$ & $r$ & $r$ & $c$ & $r$ \\
\hline Maintenance & $c$ & $\mathrm{C}$ & $c$ & $\mathrm{c}$ & $\mathrm{C}$ \\
\hline Online support & $\mathrm{C}$ & $\mathrm{C}$ & $\mathrm{c}$ & $c$ & $c$ \\
\hline Parts supply & $\mathrm{C}$ & $\mathrm{C}$ & $\mathrm{C}$ & $\mathrm{C}$ & $\mathrm{C}$ \\
\hline Upgrades & $c$ & $c$ & $\mathrm{C}$ & $\mathrm{C}$ & $\mathrm{C}$ \\
\hline
\end{tabular}

6 What is your key driver for O\&M (operation \& maintenance) of your plant? *

\begin{tabular}{|c|c|c|c|c|c|}
\hline . & $\begin{array}{l}\text { strongly } \\
\text { agree }\end{array}$ & Agree & Neutral & Disagree & $\begin{array}{l}\text { Strongly } \\
\text { disagree }\end{array}$ \\
\hline $\begin{array}{l}\text { High combined cycle } \\
\text { efficiency }\end{array}$ & $C$ & $\mathrm{C}$ & $\mathrm{C}$ & $\mathrm{C}$ & $\mathrm{C}$ \\
\hline Low NOx emission & $\mathrm{C}$ & $\mathrm{C}$ & $\mathrm{C}$ & $\mathrm{C}$ & $\mathrm{C}$ \\
\hline $\begin{array}{l}\text { Low specific cost } \\
\text { (per } K W)\end{array}$ & $\mathrm{C}$ & 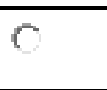 & $\mathrm{c}$ & $\mathrm{C}$ & $\mathrm{C}$ \\
\hline High availability & $\mathrm{C}$ & $\mathrm{C}$ & $\mathrm{C}$ & $\mathrm{C}$ & $\mathrm{C}$ \\
\hline Operating flexibility & $\mathrm{C}$ & $c$ & $\mathrm{c}$ & $\mathrm{C}$ & $\mathrm{C}$ \\
\hline
\end{tabular}




\begin{tabular}{|c|c|c|c|c|c|}
\hline High reliability & $\mathrm{C}$ & $c$ & $\mathrm{C}$ & $\mathrm{C}$ & $r$ \\
\hline High simple cycle & $\mathrm{C}$ & $\mathrm{C}$ & $c$ & $\mathrm{C}$ & $\mathrm{C}$ \\
\hline Cooling Technology & $\mathrm{C}$ & $\mathrm{C}$ & $\mathrm{C}$ & $\mathrm{C}$ & $\mathrm{c}$ \\
\hline Firing temperature & $\mathrm{C}$ & $\mathrm{C}$ & 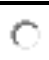 & $c$ & $r$ \\
\hline Pressure ratio & $\mathrm{C}$ & $\mathrm{C}$ & $\mathrm{C}$ & $\mathrm{C}$ & $c$ \\
\hline Mass flow & $r$ & $r$ & $r$ & $\mathrm{C}$ & $r$ \\
\hline
\end{tabular}

7 Please rank below given O\&M cost factors, in order of its importance for your plant *

\begin{tabular}{|c|c|c|c|c|c|}
\hline & $\begin{array}{l}\text { Least } \\
\text { important }\end{array}$ & $\begin{array}{l}\text { Slightly } \\
\text { important }\end{array}$ & Important & $\begin{array}{l}\text { Quite } \\
\text { important }\end{array}$ & $\begin{array}{l}\text { Very } \\
\text { important }\end{array}$ \\
\hline $\begin{array}{l}\text { Planned outage } \\
\text { frequency }\end{array}$ & 0 & $\mathrm{O}$ & $\mathrm{O}$ & 0 & 0 \\
\hline Outage duration & 0 & $\mathrm{c}$ & $c$ & $\mathrm{C}$ & 0 \\
\hline $\begin{array}{l}\text { Parts replacement } \\
\text { frequency }\end{array}$ & 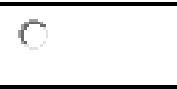 & $c$ & $c$ & $c$ & $c$ \\
\hline $\begin{array}{l}\text { Parts replacement } \\
\text { cost }\end{array}$ & 0 & $c$ & 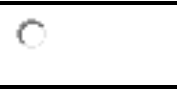 & 0 & 0 \\
\hline $\begin{array}{l}\text { Uncertainty repair / } \\
\text { maintenance }\end{array}$ & 0 & 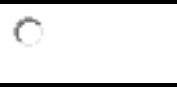 & $c$ & $\mathrm{c}$ & $c$ \\
\hline $\begin{array}{l}\text { Daily operation } \\
\text { costs } \\
\end{array}$ & 0 & $\mathrm{C}$ & $\mathrm{c}$ & 0 & 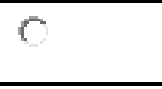 \\
\hline NOx abatement & 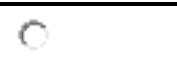 & $\mathrm{O}$ & $c$ & $\mathrm{C}$ & 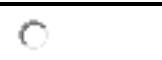 \\
\hline
\end{tabular}

8 What services BGGTS needs to improve in future *

\begin{tabular}{|c|c|c|c|c|c|}
\hline & $\begin{array}{l}\text { Strongly } \\
\text { agree }\end{array}$ & Agree & Neutral & Disagree & $\begin{array}{l}\text { Strongly } \\
\text { disagree }\end{array}$ \\
\hline $\begin{array}{l}\text { Long time surety of } \\
\text { price }\end{array}$ & $c$ & $c$ & $c$ & 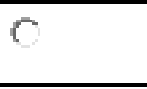 & 0 \\
\hline $\begin{array}{l}\text { Provision of high } \\
\text { quality outage } \\
\text { services }\end{array}$ & 0 & $c$ & $c$ & $\mathrm{C}$ & 0 \\
\hline $\begin{array}{l}\text { Local project } \\
\text { management \& } \\
\text { execution }\end{array}$ & 0 & 0 & 0 & 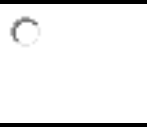 & 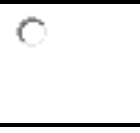 \\
\hline $\begin{array}{l}\text { Rapid supply of } \\
\text { key parts in case of } \\
\text { emergency failures }\end{array}$ & $c$ & $c$ & $c$ & $c$ & $c$ \\
\hline $\begin{array}{l}\text { Remote expert } \\
\text { monitoring of plant }\end{array}$ & 0 & 0 & $\mathrm{C}$ & 0 & 0 \\
\hline $\begin{array}{l}\text { An onsite expert to } \\
\text { provide immediate } \\
\text { advice \& } \\
\text { coordinate } \\
\text { activities }\end{array}$ & 0 & 0 & 0 & 0 & 0 \\
\hline
\end{tabular}


Research Study On After Sales Services of Gas Turbines

9 Which conversion / modification \& upgrade services are important for your plant? *

\begin{tabular}{|llllll|} 
& $\begin{array}{l}\text { Strongly } \\
\text { agree }\end{array}$ & Agree & Neutral & Disagree & $\begin{array}{l}\text { Strongly } \\
\text { disagree }\end{array}$ \\
\hline $\begin{array}{l}\text { Fuel system } \\
\text { conversions }\end{array}$ & $\mathrm{C}$ & $\mathrm{C}$ & $\mathrm{C}$ & $\mathrm{C}$ \\
\hline $\begin{array}{l}\text { Emission abatement } \\
\text { systems }\end{array}$ & $\mathrm{C}$ & $\mathrm{C}$ & $\mathrm{C}$ & $\mathrm{C}$ & $\mathrm{C}$ \\
\hline $\begin{array}{l}\text { Auxiliary system } \\
\text { modification }\end{array}$ & $\mathrm{C}$ & $\mathrm{C}$ & $\mathrm{C}$ & $\mathrm{C}$ \\
\hline $\begin{array}{l}\text { Instrumentation \& } \\
\text { control system } \\
\text { modification }\end{array}$ & $\mathrm{C}$ & $\mathrm{C}$ & $\mathrm{C}$ & $\mathrm{C}$ \\
\hline
\end{tabular}

10 Rate below given suggestions to improve services in the particular area in future *

\begin{tabular}{|c|c|c|c|c|c|}
\hline & $\begin{array}{l}\text { Strongly } \\
\text { agree }\end{array}$ & Agree & Neutral & Disagree & $\begin{array}{l}\text { Strongly } \\
\text { disagree }\end{array}$ \\
\hline $\begin{array}{l}\text { Corrosion } \\
\text { monitoring }\end{array}$ & $\mathrm{C}$ & 0 & 0 & $\mathrm{C}$ & 0 \\
\hline Air sampling & $\mathrm{C}$ & $\mathrm{C}$ & $\mathrm{C}$ & $\mathrm{C}$ & $\mathrm{C}$ \\
\hline Material testing & 0 & $c$ & 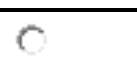 & $c$ & 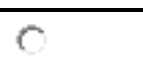 \\
\hline Site surveys & $\mathrm{C}$ & $\mathrm{C}$ & $C$ & $\mathrm{C}$ & $C$ \\
\hline $\begin{array}{l}\text { Technical advisory } \\
\text { services }\end{array}$ & $\mathrm{C}$ & $\mathrm{C}$ & $\mathrm{C}$ & $\mathrm{C}$ & $\mathrm{C}$ \\
\hline
\end{tabular}

11 What you like about BGGTS services the most? *

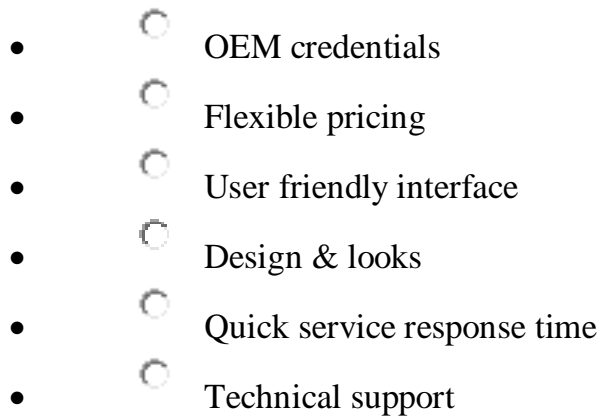

12 What are the most preferred decision making factor? (Select 1 factors) *

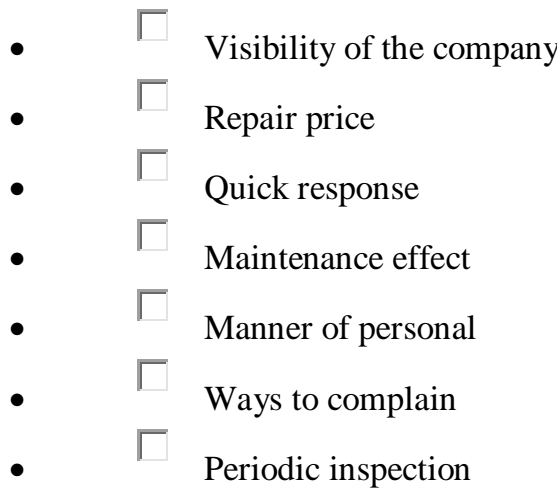


Research Study On After Sales Services of Gas Turbines

13 Rate below given factors *

\begin{tabular}{|c|c|c|c|c|c|}
\hline & $\begin{array}{l}\text { Strongly } \\
\text { agree }\end{array}$ & Agree & Neutral & Disagree & $\begin{array}{l}\text { Strongly } \\
\text { disagree }\end{array}$ \\
\hline $\begin{array}{r}\text { Your next order will be } \\
\text { to BGGTS }\end{array}$ & $C$ & $C$ & $r$ & $C$ & $C$ \\
\hline $\begin{array}{r}\text { Satisfied by services } \\
\text { delivered }\end{array}$ & $C$ & $C$ & $c$ & $C$ & $C$ \\
\hline $\begin{array}{r}\text { Services worth money } \\
\text { paid }\end{array}$ & $\mathrm{C}$ & $C$ & $C$ & $C$ & $C$ \\
\hline $\begin{array}{r}\text { BGGTS response time is } \\
\text { satisfactory }\end{array}$ & $\mathrm{C}$ & $\mathrm{C}$ & 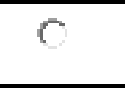 & 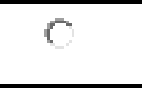 & $C$ \\
\hline $\begin{array}{r}\text { Technical support is } \\
\text { satisfactory }\end{array}$ & $C$ & 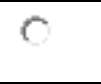 & $c$ & $C$ & $C$ \\
\hline
\end{tabular}

